Accepted for publication in APJ; JULY 1, 2021

Preprint typeset using $\mathrm{LAT}_{\mathrm{E}} \mathrm{X}$ style emulateapj v. 01/23/15

\title{
ALMA OBSERVATION OF NGC 5135: THE CIRCUMNUCLEAR CO (6-5) AND DUST CONTINUUM EMISSION AT 45 PARSEC RESOLUTION`
}

\author{
Tianwen Cao ${ }^{1,2,3,4}$, Nanyao Lu ${ }^{1}$, C. Kevin Xu ${ }^{1}$, Yinghe Zhao ${ }^{5,6,7}$, Venu Madhav Kalari ${ }^{8}$, Yu Gao ${ }^{9}$, Vassilis \\ Charmandaris ${ }^{10,11}$, Tanio Diaz Santos ${ }^{12}$, Paul van der Werf ${ }^{13}$, Chen Cao ${ }^{14,15}$, Hong Wu ${ }^{2,3}$, Hanae Inami ${ }^{16}$, \\ AARON Evans 17,18
}

Accepted for publication in APJ; July 1, 2021

\begin{abstract}
We present high-resolution $\left(0.17^{\prime \prime} \times 0.14^{\prime \prime}\right)$ Atacama Large Millimeter/submillimeter Array (ALMA) observations of the $\mathrm{CO}(6-5)$ line, and $435 \mu \mathrm{m}$ dust continuum emission within a $\sim 9^{\prime \prime} \times 9^{\prime \prime}$ area centered on the nucleus of the galaxy NGC 5135. NGC 5135 is a well-studied luminous infrared galaxy that also harbors a Compton-thick active galactic nucleus (AGN). At the achieved resolution of $48 \times 40 \mathrm{pc}$, the $\mathrm{CO}(6-5)$ and dust emissions are resolved into gas "clumps" along the symmetrical dust lanes associated with the inner stellar bar. The clumps have radii between $\sim 45-180 \mathrm{pc}$ and $\mathrm{CO}(6-5)$ line widths of $\sim 60-88 \mathrm{~km} \mathrm{~s}^{-1}$. The $\mathrm{CO}(6-5)$ to dust continuum flux ratios vary among the clumps and show an increasing trend with the $[\mathrm{Fe}$ II $] / \mathrm{Br}-\gamma$ ratios, which we interpret as evidence for supernovadriven shocked gas providing a significant contribution to the $\mathrm{CO}(6-5)$ emission. The central AGN is undetected in continuum, nor in $\mathrm{CO}(6-5)$ if its line velocity width is no less than $\sim 40 \mathrm{~km} \mathrm{~s}^{-1}$. We estimate that the AGN contributes at most $1 \%$ of the integrated $\mathrm{CO}(6-5)$ flux of $512 \pm 24 \mathrm{Jy} \mathrm{km} \mathrm{s}^{-1}$ within the ALMA field of view, which in turn accounts for $\sim 32 \%$ of the $\mathrm{CO}(6-5)$ flux of the whole galaxy.
\end{abstract}

Keywords: galaxies: active - galaxies: starbusrt - galaxies: general - galaxies: nuclei - galaxies: ISM

\section{INTRODUCTION}

${ }^{1}$ Chinese Academy of Sciences South America Center for Astronomy, National Astronomical Observatories, Chinese Academy of Sciences, Beijing 100101, China; twcao@bao.ac.cn

2 Key Laboratory of Optical Astronomy,National Astronomical Observatories, Chinese Academy of Sciences, Beijing 100101, China

${ }^{3}$ School of Astronomy and Space Science, University of Chinese Academy of Sciences, Beijing 100049, China

${ }^{4}$ Instituto de Astrofisica, Pontificia Universidad Católica de Chile,Av.Vicuña Mackenna4860, 7820436 Macul,Santiago, Chile

${ }^{5}$ Yunnan Observatories, Chinese Academy of Sciences, Kunming 650011, China

${ }^{6}$ Key Laboratory for the Structure and Evolution of Celestial Objects, Chinese Academy of Sciences,Kunming 650011, China

${ }^{7}$ Center for Astronomical Mega-Science, CAS, 20A Datun Road, Chaoyang District, Beijing 100012, China

8 Departamento de Astronoma, Universidad de Chile, Casilla 36-D, Correo Central,Santiago, Chile

${ }^{9}$ Purple Mountain Observatory/Key Lab of Radio Astronomy, 8 YuanHua Road, 210034 Nanjing, China

${ }^{10}$ Department of Physics, University of Crete, GR-71003 Heraklion, Greece

${ }^{11}$ IAASARS, National Observatory of Athens, GR-15236, Pen-teli, Greece

12 Ncleo de Astronoma de laFacultad deIngeniera, Universidad Diego Portales,Av. Ejercito Libertador 441,Santiago,Chile

13 Leiden Observatory, Leiden University, P.O. Box 9513, 2300 RA Leiden, The Netherlands

${ }^{14}$ School of Space Science and Physics, Shandong University, Weihai, Weihai, Shandong 264209, China

15 Shandong Provincial Key Laboratory of Optical Astronomy \& Solar-TerrestriaEnvironment, Weihai, Shandong 264209,China

${ }^{16}$ Univ. Lyon, Univ. Lyon1, ENS de Lyon, CNRS, Centre de Recherche Astrophysique de Lyon (CRAL) UMR 5574, 69230 Saint-Genis-Laval, France

${ }^{17}$ Department of Astronomy, University of Virginia, Charlottesville, VA 22903-2325, USA

18 National Radio Astronomy Observatory, 520 Edgemont

Road, Charlottesville, VA 22903-2475, USA

* The National Radio Astronomy Observatory is a facility of the National Science Foundation operated under cooperative agreement by Associated Universities, Inc.
Luminous infrared galaxies (LIRGs; $L_{\mathrm{IR}}^{[8-1000 \mu \mathrm{m}]} \gtrsim$ $\left.10^{11} L_{\odot}\right)$, whose space density exceeds that of optically selected starburst and Active Galactic Nucleus (AGN) host galaxies at comparable bolometric luminosities (Soifer et al. 1987), consist of isolated galaxies, galaxy pairs, interacting galaxy systems and advanced mergers (Sanders \& Mirafbel 1996; Wang et al. 2006). LIRGs in the later stages of evolution tend to contain a rich amount of molecular gas in the galaxy nuclear region (Sanders et al. 1986), and have a higher fraction of AGNs compared with less luminous galaxies (Sanders \& Mirafbel 1996). Detailed investigations of the physical properties, AGN-starburst connection, and gas inflow/outflow in representative LIRGs in the local Universe are critical to our understanding of galaxy evolution because LIRGs are the dominant contributors to the cosmic star formation (SF) at $z \gtrsim 1$ (Le Floc'h et al. 2005; Caputi et al. 2007; Magnelli et al. 2009, 2011; Gruppioni et al. 2013)

The CO emission lines from low- $J$ transitions, such as $\mathrm{CO}(1-0)$ at $2.6 \mathrm{~mm}$ and $\mathrm{CO}(2-1)$ at $1.3 \mathrm{~mm}$, have been widely used to trace the molecular gas content in LIRGs (Solomon \& Sage 1988; Sanders et al. 1991; Solomon et al. 1997; Bryant \& Scoville 1999; Gao \& Solomon 1999; Evans et al. 2002). However, based on the data taken with the SPIRE Fourier Transform Spectrometer (FTS; Griffin et al. 2010) onboard the Herschel Space Observatory (Herschel; Pilbratt et al. 2010) on a flux-limited sample of 123 LIRGs from the Great Observatories AllSky LIRGs Survey (GOALS; Armus et al. 2009), Lu et al. $(2014,2017)$ showed that the mid- $J$ CO emission (i.e., 4 $<J<10$ ) from warm and dense molecular gas correlates linearly with the star formation rate (SFR) on galactic scale for LIRGs over a wide range of $L_{\mathrm{IR}}$ and far-infrared (FIR) color. Therefore, the heating mechanism for the 
warm dense gas that gives rise to the mid- $J$ CO line emission should ultimately derive the energy from the same SF process that powers the dust emission. There is not yet a firm consensus on this heating mechanism. Apparently different heating mechanisms are favored from analyses of the CO emission line spectra of individual galaxies, including far-UV photon heating (e.g., Rigopoulou et al. 2013), heating by cosmic rays enhanced by supernovae (SNe; e.g., Bradford et al. 2003), and heating by shocks that may or may not be powered by $\mathrm{SNe}$ (e.g., Kamenetzky et al. 2012; Rangwala et al. 2011; Nikola et al. 2011; Meijerink et al. 2013; Pellegrini et al. 2013; Rosenberg et al. 2014). The X-ray photons from an AGN can heat the surrounding dense gas very effectively (e.g., Spaans \& Meijerink 2008). However, Lu et al. (2017) argued that the CO line emission associated with any AGN gas heating may peak at $J>10$. As a result, the mid- $J$ $\mathrm{CO}$ line emission is always dominated by SF.

With the Atacama Large Millimeter/submillimeter Array (ALMA; Wootten \& Thompson 2009), it is now possible to obtain high-resolution mid- $J$ CO line and dust continuum images of the nuclei of nearby LIRGs to investigate whether the Herschel results above still hold true at physical scales down to the typical size of giant molecular clouds (GMCs; i.e., $\sim 40$ pc, Kawamura et al. 2009), and whether one can rule out some of the gas heating mechanisms proposed. To this end, we have carried out a number of ALMA Band-9 observations over time, to image simultaneously the $\mathrm{CO}(6-5)$ line emission (the rest frequency $\nu_{\text {rest }}=691.473 \mathrm{GHz}$ ) and its underlying dust continuum at $435 \mu \mathrm{m}$ in the nuclear regions of a set of carefully selected, representative LIRGs from our Herschel FTS sample. The targets observed include NGC 34 (Xu et al. 2014) and NGC 1614 (Xu et al. 2015), two advanced mergers with a warm FIR color; NGC 7130 (Zhao et al. 2016) and NGC 5135 (in this paper), two well-known Seyfert galaxies with a prominent stellar bar; IC 5179 (Zhao et al. 2017), an isolated, unbarred galaxy with a compact nuclear starburst; and CGCG 049-057 with a high-surface density nuclear SF disk (Cao et al., in preparation). The linear resolutions $\left(R_{\text {linear }}\right)$ achieved range from $100 \mathrm{pc}$ in the early observation of NGC 34 to $34 \mathrm{pc}$ in the case of IC 5179. Three additional LIRGs in our FTS sample also have ALMA CO (6-5) images in the literature: $\operatorname{Arp} 220\left(R_{\text {linear }} \sim 165\right.$ pc; Wilson et al. 2014 ; Rangwala et al. 2015), IRAS $13120-5453\left(R_{\text {linear }} \sim 165\right.$ pc; Sliwa et al. 2017), and NGC 1068 ( $R_{\text {linear }} \sim 4$ pc; García-Burillo et al. 2014, 2016). ALMA CO (6-5) images also exist for two nearby, but non-LIRG galaxies: NGC 1377 (Aalto et al. 2017) and Centaurus A (Espada et al. 2017).

At a distance of $59 \mathrm{Mpc}\left(1^{\prime \prime}\right.$ corresponds to $\left.281 \mathrm{pc}\right)$ and with a fairly face-on disk, NGC 5135 is a well studied LIRG with $L_{\mathrm{IR}}=10^{11.33} L_{\odot}$ and a moderately warm FIR color of 0.54 (in terms of the 60 -to- $100 \mu \mathrm{m}$ flux density ratio; Armus et al. 2009). The galaxy not only displays a powerful circumnuclear starburst over a region of $\sim 1 \mathrm{kpc}$ in diameter (González Delgado et al. 1998; Bedregal et al. 2009) but also harbors a highly obscured Seyfert $2 \mathrm{nu}-$ cleus (Levenson et al. 2004; Phillips et al. 1983; Turner et al. 1997). It is therefore an ideal target for highresolution ALMA observations to separate the circumnuclear SF from the AGN. The $6 \mathrm{~cm}$ radio continuum emission peaks in an area $\sim 3^{\prime \prime}$ south of the nucleus based on a
Very Large Array (VLA) observation by Ulvestad \& Wilson (1989), presumably tracing the supernova remnants (SNRs) from a previous starburst. The high-resolution Hubble Space Telescope (HST) UV/optical imaging observations unveiled a large number of young star clusters, between the nucleus and the radio continuum peak (González Delgado et al. 1998), which presumably have partially cleared gas. Furthermore, the high-resolution near- and mid-infrared images (Alonso-Herrero et al. 2006; Díaz-Santos et al. 2008) show patches of strong on-going SF along, but at the downstream side of the dust lanes that are likely associated with the stellar bar (e.g. (Mulchaey \& Regan 1997)). An intermediate resolution $(R \sim 3000-4000)$, near-infrared integral-field spectroscopy (Bedregal et al. 2009) confirmed the presence of a high-excitation ionization cone centered on the AGN, based on the [Si VI] $1.96 \mu \mathrm{m}$ line emission, as well as an extended distribution of shocked gas likely powered by $\mathrm{SNe}$, based on the [Fe II] $1.46 \mu \mathrm{m}$ line. Fukazawa et al. (2011) and Singh et al. (2012) obtained broad-band (10-50 kev) X-ray spectra of NGC 5135, demonstrating that the AGN in NGC 5135 is obscured by Comptonthick material. Our ALMA imaging of NGC 5135 presented here provides for the first time the distribution and kinematics of the warm and dense molecular gas as well as the morphology of the $435 \mu \mathrm{m}$ dust emission, at a linear resolution of less than $50 \mathrm{pc}$ in the circumnuclear region of NGC 5135.

In the remainder of the paper, we describe our ALMA observation and data reduction in $\S 2$ and present our results in $\S 3$. In $\S 4$, we discuss the physical implications derived from our data on the circumnuclear SF and the role of the AGN, compare our ALMA images with existing images at other wavelengths, and comment on the most likely heating mechanism for the observed $\mathrm{CO}(6-5)$ emission. Thereby allowing us to distinguish between different heating mechanisms, and the role played by SF in giving rise to the mid-J CO emission. Finally, we summarize our results in $\S 5$. Throughout this paper, we adopt a distance of $59 \mathrm{Mpc}$ for NGC 5135 (Armus et al. 2009).

\section{OBSERVATION AND DATA REDUCTION}

The ALMA band-9 observation of NGC 5135 was carried out in the time division mode (with a velocity resolution of $\sim 6.8 \mathrm{~km} \mathrm{~s}^{-1}$ ). The four basebands (i.e., Spectral Windows; SPWs 0-3) were centered on sky frequencies of $681.975,683.736,678.243$ and $680.183 \mathrm{GHz}$, respectively, with a bandwidth of $1.875 \mathrm{GHz}$. The observation was performed with the configuration mode C34-5, using 39 12-meter antennae with baselines ranging from 21.3 to 885.6 meters. The total on-target integration time is 21.03 minutes. During the observation, the phase calibration and amplitude were monitored using J1316-3328. Additional observing details can been found in Table 1.

The data were reduced using the Common Astronomical Software Application (CASA) version 4.5 (McMullin et al. 2007). Our primary beam is $\sim 8.8^{\prime \prime}$ and the Maximum Recoverable Scale (MRS $)^{1}$ is $3.5^{\prime \prime}$. The CO (6-5) line data cube was generated using the data in the SPW covering the sky frequency range of 680.975 to $682.975 \mathrm{GHz}$. The continuum was estimated by combining the data from the other three SPWs. The calibrated images were cleaned using the Briggs weighting (with the parameter "robust" set to 0.5 ). The resulting synthesized 
beams are nearly identical between the continuum and the line emission and have a full width at half maximum (FWHM) size of $\sim 0.17^{\prime \prime} \times 0.14^{\prime \prime}$ (equivalent to $48 \times 40 \mathrm{pc}$ at the distance to NGC 5135), with the major-axis position angle (north to east) at $111^{\circ}$. All our analyses in this paper use the data after the primary beam correction, whereas all of the figures (except Fig. 6) were produced using data prior to the primary beam correction.

The final spectral cube has a channel width equivalent to $13.5 \mathrm{~km} \mathrm{~s}^{-1}$ in velocity. The channel noise $\left(\sigma_{\mathrm{ch}}\right)$ is on the order of $18 \mathrm{mJy}$ beam $^{-1}$. The total CO (6-5) flux image, as an integration over the barycentric velocities from 3,971 to $4,157 \mathrm{~km} \mathrm{~s}^{-1}$, has an r.m.s. noise of $\sim 1.2$ Jy beam ${ }^{-1} \mathrm{~km} \mathrm{~s}^{-1}$. The continuum image has a noise of $2.2 \mathrm{mJy}_{\text {beam }}{ }^{-1}$. All these noise measurements were done on the data without the primary beam correction. The ALMA absolute flux calibration is estimated to be good to $\sim 10 \%$. The astrometric accuracy is better than $0.01^{\prime \prime}$.

\section{RESULTS}

\subsection{CO (6-5) Line Emission}

The four panels in Fig. 1 show respectively the images of the total $\mathrm{CO}(6-5)$ emission integrated between the observed velocities of 3971 and $4157 \mathrm{~km} \mathrm{~s}^{-1}$, the $435 \mu \mathrm{m}$ continuum, the velocity field (i.e., moment 1 ), and velocity dispersion map (moment 2). Each image roughly covers the ALMA primary beam. The contours overlaid in Fig. 1a and 1b refer to the same total CO (6-5) emission and start at $\mathrm{S} / \mathrm{N}=3$.

The $\mathrm{CO}(6-5)$ emission detected at $\mathrm{S} / \mathrm{N}>3$ appears clumpy and is confined to a few discrete regions along two spiral arm-like features corresponding spatially to the dust lanes seen in the UV and optical (e.g., Muñoz Marín et al. 2007). We mark four separate regions, namely $(a, b, c, d)$ which are compact and concentrated in the intergrated $\mathrm{CO}(6-5)$ map. The region $d$ appears more diffuse compared with the other three. The total $\mathrm{CO}(6-5)$ flux from the combined four regions in Fig. 1a is $512 \pm 24 \mathrm{Jy} \mathrm{km} \mathrm{s}^{-1}$. With a much larger beam of $\sim 31^{\prime \prime}$, the Herschel/FTS observation gives a $\mathrm{CO}(6-5)$ flux of $1,617 \mathrm{Jy} \mathrm{km} \mathrm{s}^{-1}(\mathrm{Lu}$ et al. 2017). Therefore the clump regions in Fig. 1a together account for $\sim 32 \%$ of the total CO (6-5) flux of the galaxy. The "missing" line flux could be due to a combination of the line emission outside the ALMA field of view, or possible faint emission at peak surface brightness below our 3- $\sigma$ (i.e., 3.6 Jy beam ${ }^{-1} \mathrm{~km} \mathrm{~s}^{-1}$ ) detection limit, or resolved out on larger scales. We analyze individual clumps in more detail in $\S 4.2$.

We set the threshold at $4-\sigma_{\mathrm{ch}}$, where $\sigma_{\mathrm{ch}}$ is the r.m.s noise per frequency channel, to obtain the moment 1 and 2 maps. To reveal the kinematics better, the moment 1 and 2 maps, shown respectively in Fig. 1c and 1d, were based on the uv-taper image (the details about the uvtaper image are presented in the last paragraph of this subsection). The velocity scale in Fig. 1c was calculated using the formula $\nu_{\text {obs }}=\nu_{\text {rest }}(1-V / c)$, where $\nu_{\text {obs }}$ is the observed $\mathrm{CO}(6-5)$ line frequency, $c$ the speed of light, and $V$ the velocity to calculate. The line velocity ranges

\footnotetext{
${ }^{1}$ https://science.nrao.edu/science/videos/largest-angular-scaleand-maximum-recoverable-scale
}

from 3992 to $4140 \mathrm{~km} \mathrm{~s}^{-1}$. Fig. 1d shows that the lineof-sight velocity dispersion ranges from 10 to $40 \mathrm{~km} \mathrm{~s}^{-1}$, using only those pixels with $\mathrm{S} / \mathrm{N}>4-\sigma_{\mathrm{ch}}$. The overall kinematic pattern can also be seen in the channel maps displayed in Fig. 2, where the contours from an individual channel of width $13.5 \mathrm{~km} \mathrm{~s}^{-1}$ are overlaid on the grayscale image of the total $\mathrm{CO}(6-5)$ flux map shown in Fig. 1a. While the regions $a, c, d$ are mainly confined within a velocity range of 3992 to $4073 \mathrm{~km} \mathrm{~s}^{-1}$, the region $b$ has a range between 4046 and $4127 \mathrm{~km} \mathrm{~s}^{-1}$. This suggests that the observed velocity pattern is not dominated by a simple rotation within the galaxy disk. In the channel maps, some $\mathrm{CO}$ (6-5) clumps break down into smaller clumps (or clouds) in some velocity channels, e.g., the region $a$ in the channel centered at $V=4073 \mathrm{~km} \mathrm{~s}^{-1}$. These clouds have sizes $<50 \mathrm{pc}$.

In Fig. $3 \mathrm{a}$ and 3b, we reproduced the same images as in Fig. $1 \mathrm{a}$ and $1 \mathrm{~b}$, respectively, but with a larger effective beam of $0.4^{\prime \prime} \times 0.4^{\prime \prime}$ (equivalent to $112 \times 112$ pc) by applying an uv-taper (with the parameter "outertaper" $\left.=0.4^{\prime \prime}\right)$ to our uv data before imaging. The peak signal of the region $d$ is higher than $4-\sigma$ ( $\sigma=$ $8 \mathrm{Jy}$ beam $^{-1} \mathrm{~km} \mathrm{~s}^{-1}$ ). The total flux of the four regions combined, as defined in Fig. 1, is $841 \pm 45 \mathrm{Jy} \mathrm{km} \mathrm{s}^{-1}$. This flux equals 1.6 times the flux from the original ALMA image, and is $~ 52 \%$ of the total flux measured by Herschel, confirming that there exists some more diffuse or lower surface brightness CO (6-5) emission within the region of Fig. 1a.

\subsection{Dust Continuum Emission}

As shown in Fig. 3b, the continuum at $435 \mu \mathrm{m}$ generally coincides with the $\mathrm{CO}(6-5)$ line emission in regions $a$ and $c$ at scales of $0.4^{\prime \prime}$ (equivalent to $112 \mathrm{pc}$ ), which corresponds to an angular size of $114 \mathrm{pc}$ at the distance of NGC 5135. This is consistent with the findings in the other LIRGs we imaged in $\mathrm{CO}(6-5)$, i.e., NGC 34, NGC 1614 and NGC 7130 and IC 5179 (Xu et al. 2014, 2015; Zhao et al. 2016, 2017), i.e., at scales $\gtrsim$ $100 \mathrm{pc}$, there is a good spatial correspondence between the $\mathrm{CO}(6-5)$ line and its underlying continuum emissions.

However, at scales significantly smaller than $100 \mathrm{pc}$, there are apparent offsets between the local peaks of the line and continuum emissions in Fig. 1b. This small-scale offset between the line and continuum emissions is also seen in the LIRGs of moderately high nuclear gas surface densities, e.g., IC 5179 (at linear resolution $R_{\text {linear }} \approx$ $34 \mathrm{pc}$; Zhao et al. 2017$)$ and NGC $7130\left(R_{\text {linear }} \approx 70 \mathrm{pc}\right.$ $\times 40$ pc; Zhao et al. 2016). Furthermore, in both Fig. 1b and $3 \mathrm{~b}$, the dust continuum is unusually weak relative to the line emission in the regions $b$ and $d$. As argued in Zhao et al. (2016), these differences between the line and dust continuum emissions at small scales can only be understood if the gas and dust are heated by different mechanisms. We discuss this in more detail in $§ 4.3$.

By combining the four regions in Fig. 1b, we derived a total flux of $181 \pm 25$ mJy for the $435 \mu \mathrm{m}$ continuum emission. This flux would be 1.6 times higher if we had derived it from the same regions in Fig. 3b.

\section{ANALYSIS AND DISCUSSION}

\subsection{The Central AGN}


The AGN position can be constrained by the peak of the [Si VI] line emission at $1.96 \mu \mathrm{m}$ in a ground-based observation (Bedregal et al. 2009) and by the peak of the hard X-ray (4-8 keV) emission detected with Chandra (Levenson et al. 2004). The estimated astrometric uncertainty associated with either of these images is on the order of $0.5^{\prime \prime}$. The VLA $6 \mathrm{~cm}$ radio continuum image of Ulvestad \& Wilson (1989) has a modest resolution of $0.91^{\prime \prime} \times 0.60^{\prime \prime}$ and an astrometric accuracy of $0.3^{\prime \prime}$. We overlaid our $\mathrm{CO}(6-5)$ contours from Fig. 1a on this radio continuum image in Fig. 4a. As already stated in Sec. 1, the main peak of the $6 \mathrm{~cm}$ emission is $\sim 3^{\prime \prime}$ south of the galaxy nucleus, spatially coincident with the peak of the broad (FWHM $=\sim 513 \mathrm{~km} \mathrm{~s}^{-1}$ ) [FeII] emission (Bedregal et al. 2009). However, there is a minor radio emission peak $(10 \sigma)$ near the anticipated AGN position. We take the position of this radio peak (R.A. $=13^{h} 25^{m} 44^{s} .02$, Dec. $=-29^{\circ} 50^{\prime} 00^{\prime \prime} .4$; J2000) as the AGN location (i.e., marked by the white cross), with a positional uncertainty of $0.3-0.5^{\prime \prime}$. In Fig. $4 \mathrm{~b}$, we overlaid the same CO (6-5) contours on the hard X-ray emission.

As shown in Fig. 1a, the $\mathrm{CO}\left(6^{-5}\right)$ emission is undetected at $4-\sigma$ level at the AGN position (i.e., integrated over the velocity range of $186 \mathrm{~km} \mathrm{~s}^{-1}$ ). This would hold true even if we had lowered the detection threshold to 3- $\sigma$. We assume the AGN-related CO (6-5) emission is confined to an area smaller than our ALMA beam size $(\sim 48 \times 40 \mathrm{pc})$, then the $3-\sigma$ flux upper limit is equal to $3 \times\left(1.2 \mathrm{Jy} \mathrm{km} \mathrm{s}^{-1}\right)=3.6 \mathrm{Jy} \mathrm{km} \mathrm{s}^{-1}(\sigma=$ 1.2 $\left.\mathrm{Jy} \mathrm{beam}^{-1} \mathrm{~km} \mathrm{~s}^{-1}\right)$.

However, an apparent narrow emission feature at the AGN location is seen at 3-4 $\sigma$ significance over only two velocity channels (i.e., $\mathrm{V}=4019.0$ and $4032.5 \mathrm{~km} \mathrm{~s}^{-1}$ ). The image summed over these two velocity channels is presented in Fig. 5a, which shows a peak surface brightness of $33 \mathrm{mJy}^{\mathrm{beam}}{ }^{-1}$ (at $5-\sigma$ significance). The spectrum in Fig. 5b is extracted from a circular aperture of radius $=0.4^{\prime \prime}(=2.5$ times the FWHM of the 3 $\sigma$ surface brightness of the emission in Fig. 5a). Its narrow velocity width of $\sim 40 \mathrm{~km} \mathrm{~s}^{-1}$ makes it unlikely that this signal is physically associated with the AGN. Nevertheless, considering that the CO (6-5) emission associated with the gas torus of the AGN in NGC 1068 is observed to have only a modest velocity width of $\sim 80 \mathrm{~km} \mathrm{~s}^{-1}$ (García-Burillo et al. 2016), we defer to a future observation of higher angular resolution to firmly conclude the reality of this narrow $\mathrm{CO}(6-5)$ emission. Flux-wise, the narrow $\mathrm{CO}(6-5)$ emission in Fig. 5b has a flux of $1.7 \mathrm{Jy} \mathrm{km} \mathrm{s}^{-1}$, which is smaller than the $3-\sigma$ flux upper limit of $3.6 \mathrm{Jy}_{\mathrm{km} \mathrm{s}}{ }^{-1}$ derived above. Therefore, we conclude that the AGN in NGC 5135 contributes at most $1 \%$ of the $\mathrm{CO}(6-5)$ flux observed within the ALMA field of view. This is consistent with the Herschel finding that the mid- $J$ CO line emission in LIRGs is mainly associated with SF regardless of whether there is an AGN or not (Lu et al. 2017). The fractional contribution of the AGN to the bolometric luminosity of NGC 5135 is about $(24 \pm 6) \%$ (Díaz-Santos et al. 2017). The AGN in NGC 5135 is heavily obscured; the surrounding gas could be heated to a very high temperature by the X-rays associated with the AGN, resulting in a CO spectral line distribution that peaks $J>10$ (Spaans \& Meijerink 2008). Such a scenario seems to be the case in the Seyfert galaxy
NGC 1068: While the high-resolution ALMA imaging shows that the vast majority of the nuclear CO (6-5) emission is associated with the compact circumnuclear ring of SF at a radius of $\sim 100$ pc (García-Burillo et al. 2016), the total nuclear CO emission line spectrum has a distinct component that peaks at $J \sim 16$ (Spinoglio et al. 2012). This hot spectral component of the CO emission is presumably due to the AGN in NGC 1068. The central AGN in NGC 5135 is bright in terms of the 1-0 S(1) $2.12 \mu \mathrm{m} \mathrm{H}_{2}$ ro-vibrational line (Bedregal et al. 2009) associated with warm molecular gas. Although this line could be excited by different physical processes, including UV-fluorescence (photons); shock fronts (collisions) and X-ray illumination, Bedregal et al. (2009) argued that the excited near-IR $\mathrm{H}_{2}$ emission is mainly caused by X-ray illumination in the AGN region of NGC 5135 . Such an X-ray dominant scenario is also favored based on non-detection of the $\mathrm{CO}(6-5)$ emission here.

The $435 \mu \mathrm{m}$ dust continuum is also undetected at the AGN position, with the $3-\sigma$ flux upper limit equal to $5.4 \mathrm{mJy}\left(\sigma=1.8 \mathrm{mJy}^{\mathrm{beam}}{ }^{-1}\right.$; the same method used for deriving the $\mathrm{CO}(6-5)$ flux upper limit). We compare this flux upper limit with the expected $435 \mu \mathrm{m}$ continuum flux from an average infrared spectral energy distribution (SED) appropriate for the AGNs of X-ray luminosities comparable to that of NGC 5135: The intrinsic $2.0-10 \mathrm{keV}$ X-ray luminosity of NGC 5135 is $\sim 1.8 \times 10^{43}$ $\operatorname{ergs}^{-1}$ (Singh et al. 2012). We therefore used the infrared AGN SED for $L_{2.0-10 \mathrm{keV}}>10^{42.9} \mathrm{erg} \mathrm{s}^{-1}$ in Mullaney et al. (2011) and anchored it at the $12 \mu \mathrm{m}$ luminosity of NGC 5135 estimated from the X-ray and mid-IR correlation given in Asmus et al. (2015). This derived SED is shown in Fig. 6, along with two continuum flux upper limits (at $3 \sigma$ ) at 435 and $1,300 \mu \mathrm{m}$ based on the ALMA observation. The latter continuum flux upper limit was estimated from an archival ALMA Band-6 observation (Project 2013.1.00243.S; PI: L. Colina). This plot suggests that the ALMA data points are consistent with what is expected from the typical infrared SED for AGNs like NGC 5135.

\subsection{Properties of Molecular Gas Clumps}

Several clumpy features are resolved in the CO (6-5) image shown in Fig. 1a. The resolved clump sizes of $\sim 100$ pc are comparable with or larger than the beam size. For other (U)LIRGS, such clumpy features are traced more commonly by low-J CO or isotopologues, owing to the difficulty in observing dense tracers. However, it is more appropriate to analyse the properties of compact clumpy structures as seen in Fig. 1a using denser gas tracers rather than the diffuse gas traced by low-J CO observation. Dense gas tracers, such as CS (2-1), HCN (1-0) and CO (6-5) usually trace embedded cloud clumps or cores within a more extended distribution of CO emission (Leroy et al. 2015; Sakamoto et al. 2011; Rosolowsky \& Blitz 2005), and therefore are very useful for studying the dense, embedded star-forming structures within a much larger molecular region.

In Fig. 1a, the $\mathrm{CO}(6-5)$ emission peaks are resolved into separate clumps at $\mathrm{S} / \mathrm{N}=4$, labelled as $a 1, a 2, a 3$, $a 4, a 5, b, c$ and $d$. For each clump, we list in Table 2 a number of parameters derived from the image in Fig. 1a for all the clumps except for the clump $d$. At the resolution of Fig. 1a, the clump $d$ is detected only at $\mathrm{S} / \mathrm{N}=$ 
3 and appears to be quite diffuse. We therefore derived its parameters from the image in Fig. 3a, which has a coarser resolution of $112 \times 112 \mathrm{pc}$.

The size of a clump is specified by its FWHM major and minor axes plus the major axis position angle (PA), which are respectively given in Columns (2) and (3) of Table 2. These were derived from a $2 \mathrm{~d}$ Gaussian fit to the clump intensity map. For the blended clumps ( $a 4$ and $a 5$ ), we segmented the cloud into sub-clouds employing a variant of the CLUMPFIND algorithm (Williams et al. 1994). We divided the blended clumps by the half distance of two peaks and measure their parameters. We also calculated the effective clump radius $R$, following Solomon et al. (1987). After the deconvolution with the appropriate ALMA beam (using the CASA function "deconvolvefrombeam"), the radii range from 45 to $180 \mathrm{pc}$ for the clumps (see Table 2, Column (4)).

We also extracted the 1d spectrum for each clump within an elliptical aperture with radii of (major and minor axes (FWHM)) as the oval areas marked in Fig. 1a. The resulting spectra are plotted for all the clumps in Fig. 7. Using the same elliptical aperture, we derived the integrated $\mathrm{CO}(6-5)$ flux from the clump intensity map and $435 \mu \mathrm{m}$ continuum flux density from continuum map. The line central velocity, the line velocity width $\Delta V_{\mathrm{FWHM}}$, the integrated $\mathrm{CO}(6-5)$ flux and $435 \mu \mathrm{m}$ continuum flux density given respectively in Columns (5), $(6),(8)$ and (9) in Table 2 . The resulting $\Delta V_{\text {FWHM }}$ ranges from 60 to $88 \mathrm{~km} \mathrm{~s}^{-1}$.

We also estimated the Virial and molecular gas masses for each clump. Following Larson (1981); Heyer et al. (2009), the Virial mass $\left(M_{v i r}\right)$ is estimated as

$$
M_{\mathrm{vir}} / M_{\odot}=\frac{5\left(\Delta V_{\mathrm{FWHM}} / \mathrm{km} \mathrm{s}^{-1}\right)^{2} R / \mathrm{pc}}{G},
$$

where $R$ is simply the effective clump radius and $\Delta V_{\mathrm{FWHM}}$ refers to the value of after deconvolution with channel width $13.5 \mathrm{~km} \mathrm{~s}^{-1}$ and a possible contribution of about $10 \mathrm{~km} \mathrm{~s}^{-1}$ from the disk rotation is further removed. (This correction amount was set to the mean velocity change over the size of one ALMA beam by examining the P-V plots of all the clumps. In the Following, the $\Delta V_{\text {FWHM }}$ which we have used in Fig. 8 and 9 are those corrected ones.) The derived Virial masses, shown in Column (10) of Table 2, rage from $\sim 7$ to $60 \times 10^{7} M_{\odot}$.

We estimated the molecular gas mass of a clump, $M_{\text {mol }}$, using the $850 \mu \mathrm{m}$ continuum flux density-based formula in Scoville et al. (2016) by converting the observed $435 \mu \mathrm{m}$ flux density to that at $850 \mu \mathrm{m}$ assuming a dust temperature of the $25 \mathrm{~K}$ :

$$
\begin{aligned}
L_{\nu_{850} \mu \mathrm{m}} & =1.19 \times 10^{27} \times S_{\nu} / \mathrm{Jy} \times \frac{\left(\nu_{850} \mu \mathrm{m}\right)^{3.8}}{\nu_{\text {obs }}(1+z)} \times \frac{\left(d_{L} / \mathrm{Mpc}\right)^{2}}{1+z} \\
& \times \frac{\Gamma_{\mathrm{RJ}}\left(25, \nu_{850} \mu \mathrm{m}, 0\right)}{\Gamma_{\mathrm{RJ}}\left(25, \nu_{\mathrm{obs}}, z\right)} /\left(\mathrm{erg} \mathrm{s}^{-1} \mathrm{~Hz}^{-1}\right)
\end{aligned}
$$

and

$$
\alpha_{\nu}=L_{\nu_{850} \mu \mathrm{m}} / M_{\mathrm{mol}}=6.7 \pm 1.7 \times 10^{19} /\left(\operatorname{erg~s}^{-1} \mathrm{~Hz}^{-1} \mathrm{M}_{\odot}^{-1}\right)
$$

Where $\Gamma_{\mathrm{RJ}}\left(T_{\mathrm{d}}, \nu, z\right)=\frac{h \nu / k T_{\mathrm{d}}}{e^{h \nu / k T_{\mathrm{d}}-1}}$ and the $S_{\nu}$ is the dust emission flux density. As the $435 \mu \mathrm{m}$ flux density shown in Table 2 is measured within FWHM (diameter), it should only account for about $58 \%$ of the total flux. Thus, we multiply the flux density by 1.731 to estimate the total flux. For the gas clumps in the nuclear region of NGC 5135, $T_{d}$ could be warmer. In this case, the calculated $M_{\text {mol }}$ would be overestimated by roughly a factor of $T_{d} /(25 \mathrm{~K})$. The resulting $M_{\mathrm{mol}}$, given in Column (11) of Table 2 , ranges from $\sim 1$ to $10 \times 10^{8} M_{\odot}$.

To check the molecular gas mass, we derived the molecular gas mass from the CO flux. We chose the conversion factor $\alpha_{\mathrm{CO}}=0.8 \mathrm{M}_{\odot}\left(\mathrm{K} \mathrm{km} \mathrm{s}^{-1} \mathrm{pc}^{2}\right)^{-1}$ (Downes \& Solomon 1998). The CO $(6-5) / C O(1-0)$ is about 2 (this will be explained in Sec. 4.3.1). The molecular gas mass derived this way is consistent with the one derived from the dust continuum as shown in Column (12) of Table 2.

Table 2 shows that all clumps have $M_{\text {mol }}>M_{\text {vir }}$ except for the clumps $b$ and $d$. The clumps in the former category (hereafter referred to as Category (i)) are likely to be self-gravitationally bound or even undergoing initial collapse. On the other hand, the clumps $b$ and $d$ in the other category (hereafter Category (ii)) would require external pressure to remain bound. Interestingly, the Category (ii) clumps are far away from on-going SF activity and also show significantly higher CO (6-5)-todust continuum flux ratios (cf. Table 2, Column (13)) than the clumps in Category (i).

We can also compare the clumps in NGC 5135 with the molecular gas clouds in other galaxies. Fig. 8 is a plot of the cloud (FWHM) velocity dispersion as a function of the cloud radius for the clumps in NGC 5135 as well as discrete clouds in the center of the Milky Way, nearby spiral galaxies, and two starburst galaxies NGC 253 and IC 5179. In comparison to the molecular clouds over the disks of nearby normal galaxies, the clouds in the Milky Way center observed by Oka et al. (2001) have a larger line width, but a smaller size. In contrast, the clouds in the starburst galaxy NGC 253 (Leroy et al. 2015) and the LIRG IC 5179 (Zhao et al. 2017) show both larger sizes and broader line widths than clouds in the Milky Way center, but generally following the lines of equal gas surface density for the case of Virialized clouds. The clouds in the nuclear region of NGC 5135 are characterized by still larger sizes and line widths.

Fig. 9 is a plot of the parameter, $\Delta V_{\mathrm{FWHM}}^{2} / R$, as a function of molecular gas mass surface density $\Sigma$ for the same data set as in Fig. 8. The thick diagonal line shows the locus of Virialized clouds. For bound clouds clearly lying above this line, the cloud velocity width is likely a manifestation of some external pressure. The dashed curves in Fig. 9, taken from Field et al. (2011), indicate the relationship between $\Delta V_{\mathrm{FWHM}}^{2} / R$ and $\Sigma$ for a varying external pressure. The Category (i) gas clumps in NGC 5135 lie around the line tracing the Virial equilibrium. In contrast, the two Category (ii) clumps are clearly located above the Virial equilibrium, and require external pressure of the order of $10^{8} \mathrm{~cm}^{-3} \mathrm{~K}$ in order to remain bound.

\section{3. $C O(6-5)$ Emission}

\subsubsection{CO (6-5) Emission to Continuum Ratio}

On galaxy scale, the ratio of the $\mathrm{CO}(6-5)$ line luminosity, $L_{\mathrm{CO}(6-5)}$, to $L_{\mathrm{IR}}$ varies only by up to $30 \%$ among lo- 
cal LIRGs and shows little dependence on $L_{\mathrm{IR}}$ or the FIR color (Lu et al. 2014, 2017). This strongly requires that the energy sources for both the $\mathrm{CO}(6-5)$ and the dust emissions are ultimately tied to the same SF process. This narrows down the candidate heating mechanisms for the $\mathrm{CO}(6-5)$ emission to fewer choices, including photon heating in the photon dominant regions (PDRs) around young massive stars and SN-powered shock heating.

Our recent ALMA observation of nearby LIRGs show that, on scales of $100 \mathrm{pc}$ or less, local peaks of the CO (6-5) emission do not always have corresponding peaks of the $435 \mu \mathrm{m}$ dust continuum emission. Such examples include NGC 7130 (Zhao et al. 2016), IC 5179 (Zhao et al. 2017) and the case of NGC 5135 shown here. Under the assumption of a constant dust-to-gas abundance ratio, the spatial peaks of the two emissions should follow each other if both the dust and $\mathrm{CO}(6-5)$ emissions are related to the same photon heating. This finding therefore favors the SNe-powered shock gas heating scenario for the $\mathrm{CO}(6-5)$ emission. Here we investigate further this subject in the case of NGC 5135 .

As shown in Column (13) of Table 2, the $\mathrm{CO}(6-5)$ flux to the $435 \mu \mathrm{m}$ continuum flux density ratio, $R_{\mathrm{CO} / \text { cont }}$, varies among the $\mathrm{CO}(6-5)$ clumps. Further more, while the Category (i) clumps satisfy $600 \lesssim$ $R_{\mathrm{CO} / \text { cont }} \lesssim 1800 \mathrm{~km} \mathrm{~s}^{-1}$, the two Category (ii) clumps have $R_{\mathrm{CO} / \text { cont }}>4,000 \mathrm{~km} \mathrm{~s}^{-1}$. It is evident in Table 2 , the higher $R_{\mathrm{CO} / \text { cont }}$ values associated with the Category (ii) clumps are mostly due to the unusually faint dust continuum emission at $435 \mu \mathrm{m}$. One can express

$$
R_{\mathrm{CO} / \mathrm{cont}} \propto\left(f_{\mathrm{CO}(6-5)} / f_{\mathrm{CO}(1-0)}\right)\left(M_{\text {gas }} / M_{\mathrm{dust}}\right) T_{\mathrm{d}}^{-1},
$$

where we have assumed the $\mathrm{CO}(1-0)$ flux, $f_{\mathrm{CO}(1-0)}$, scales with the molecular gas mass $M_{\text {gas }}$. This shows that a higher $R_{\mathrm{CO} / \text { cont }}$ can stem from either a hotter $\mathrm{CO}$ gas or/and a cooler dust temperature. In the nuclear region of NGC 5135, the variation of $T_{d}$ is limited to, perhaps, a factor of 3 (i.e., from 15 to $50 \mathrm{~K}$ ) at most. The observed variation of $R_{\mathrm{CO} / \text { dust }}$ is a factor of $\sim 5$ in Table 2, mainly between the two clump categories. A comparable variation is also seen in the case of IC 5179 (Zhao et al. 2017). Therefore, it requires a modest variation of a factor of 2 or so in $f_{\mathrm{CO}(6-5)} / f_{\mathrm{CO}(1-0)}$ in order to explain the observation. If the $\mathrm{CO}(6-5)$ emission is associated with SNe-shock heating, the ideal location for a higher $R_{\mathrm{CO} / \text { cont }}$ ratio is where massive $\mathrm{O}$ star formation has ended while SNe activity is still strong, a scenario we discussed in the case of NGC 7130 (Zhao et al. 2016). A necessary condition for the validity of this scenario is that some dense gas can survive the massive star formation, which might be possible in dense and clumpy ISM.

\subsection{2. $\mathrm{CO}(6-5)$ Emission and Current $S F$}

In Fig. 10, the black contours of the integrated $\mathrm{CO}(6-5)$ line emission are overlaid on a ground-based $8.7 \mu \mathrm{m}$ image (Díaz-Santos et al. 2008) on the left side, and on an HST Pa- $\alpha$ image (Alonso-Herrero et al. 2006) on the right. In both plots, we also show the lowresolution Chandra $0.4-8 \mathrm{keV}$ broadband X-ray emission (Levenson et al. 2004) in red contours. This X-ray emission is mostly associated with a hot, ionized gas powered by SNRs (Levenson et al 2004; Colina et al. 2012).
The $8.7 \mu \mathrm{m}$ image is dominated by the mid-IR emission bands from the so-called Polycyclic Aromatic Hydrocarbons (PAHs). The Pa- $\alpha$ emission is caused by the ionizing $\mathrm{UV}$ radiation from massive $\mathrm{O}$ stars and is regarded as a reasonable tracer of the on-going SF activity (timescale $\sim 10 \mathrm{Myr})$. The PAH emission traces SF of 10 times longer time scale $(\sim 100 \mathrm{Myr})$, as PAH molecules are mostly heated by non-ionizing B stars (Díaz-Santos et al. 2008). As shown in Fig. 10, the PAH and Pa- $\alpha$ emissions show similar surface brightness distributions. In contrast, the overall spatial morphology of the $\mathrm{CO}(6-5)$ emission appears to be different from that of either the $\mathrm{PAH}$ or the $\mathrm{Pa}-\alpha$ emission. However, the Category (i) CO (6-5) clumps are all relatively close to local emission peaks of the $\mathrm{PAH}$ or $\mathrm{Pa}-\alpha$ emission whereas the Category (ii) $\mathrm{CO}(6-5)$ clumps are significantly farther away from any bright $\mathrm{PAH}$ or $\mathrm{Pa}-\alpha$ peak. Therefore, it is reasonable to expect a much weaker far-UV radiation intensity at the location of each Category (ii) clump. This naturally explains why the dust emission is unusually faint at each of the Category (ii) clumps.

\subsection{Possible Heating Scenarios for CO (6-5) Emission \\ 4.4.1. SN-powered Shock Heating Scenario}

With an integral field spectrograph, Colina et al. (2012) measured the intensity and velocity fields of both the $\mathrm{Br}-\gamma$ and the [Fe II] $1.64 \mu \mathrm{m}$ emission lines in the nuclear region of NGC 5135. While the Br- $\gamma$ traces the current star formation, the [Fe II] line emission is regarded as a particularly good tracer of SNRs (Greenhouse et al. 1991). In Fig. 11, we show a plot of the [Fe II]-to-Br- $\gamma$ line ratio versus the $\mathrm{CO}$ (6-5)-to-continuum flux density ratio for all the clumps listed in Table 2, except for clump $d$, which is located outside the field of view of the [Fe II] observation. We also indicated the typical [Fe II]-to-Br- $\gamma$ line ratios for different astrophysical objects, taken from Falcón-Barroso et al. (2014). Note that the line ratio range shown for Seferts is largely irrelevant here as our molecular clouds are all located far away from the AGN.

A number of studies have attempted to identify the physical causes behind the observed variations of the [Fe II]-to-Br- $\gamma$ line ratio (e.g., Alonso-Herrero et al. 1997; Moorwood \& Oliva 1988; Mouri et al. 1990, 1993; Greenhouse et al. 1991; Rodríguez-Ardila et al. 2004, 2005; Ramos Almeida et al. 2006, 2009; Riffel et al. 2013; Falcón-Barroso et al. 2014). From these studies, two main conditions for an enhanced [Fe II] emission relative to a Hydrogen recommbination line emission emerge: (a) Presence of shocked gas and (b) favorable environment for the gas-phase $\mathrm{Fe}$ to be abudant in the form of $\mathrm{Fe}^{+}$. Iron is normally depleted onto grains in the interstellar gas phase, so fast shocks, such as those associated with $\mathrm{SNe}$, can cause grain destructions and therefore enrich gas-phase Fe abundance. Another important prerequisite for a strong [Fe II] line is an ionization field in favor of $\mathrm{Fe}^{+}$. Given the low ionization potential of $\mathrm{Fe}^{+}(16.2 \mathrm{eV})$, most of $\mathrm{Fe}$ is in higher ionization states in HII regions. In comparison, partially ionized gas in SNRs and $\mathrm{Fe}^{+}$is believed to be abundant (Moorwood \& Oliva 1988). The collisional exitation with electrons could therefore make the [Fe II] line much brighter in SNRs.

The [Fe II $]$-to-Br- $\gamma$ line ratios for the Category (i) clumps in NGC5135 are around 3, which is just out- 
side the upper tip of the range for Galactic Hir regions. These ratios are slightly higher than those seen in the nuclear star-forming regions in the Seyfert galaxy NGC 613 (Falcón-Barroso et al. 2014), suggesting some mild enhancement in the $[\mathrm{Fe} \mathrm{II}]$ line emission for the gas clouds in NGC 5135. In comparison, this line ratio for the Category (ii) clump $b$ equals 6.5 , implying a factor of 2 further enhancement in the relative [Fe II] emission from the Category (i) clouds. It is not surprising for the observed line ratio of the Category (ii) cloud to be smaller than the typical values seen in Galactic SNRs because we are averaging over a much larger area than the typical size of Galactic SNRs and also because there is still lowsurface brightness star forming activity near the cloud (see Fig. 10). The observed trend in Fig. 11 indicates that the same SNe shocks are likely to play a positive role in the observed variations in both $[\mathrm{Fe} \mathrm{II}] / \mathrm{Br}-\gamma$ and CO (6-5)/dust ratios.

Additional evidence in favor of the SNe-shock heating scenario for the CO (6-5) emission in NGC 5135 includes (a) the prevailing X-ray emission from the hot, ionized gas excited via SN shocks (Colina et al. 2012); and (b) that there is a very good velocity field correspondence between the $\mathrm{CO}(6-5)$ clumps and that of the underlying [Fe II] emission: the nominal $\mathrm{CO}(6-5)$ and [Fe II] line velocity offset varies around the mean of $-142 \mathrm{~km} \mathrm{~s}^{-1}$ by only a few $\mathrm{km} \mathrm{s}^{-1}$ among the clumps. (Note that the mean velocity field difference is likely a result of the different velocity reference frames adopted.) This velocity correspondence suggests that the warm CO gas and the shocked/ionized gas are reasonably well mixed with each other in space and velocity field. Another independent evidence in favor of the SNe-shock heating scenario is the global tight correlation between the IR dust emission and the mid-J CO line emission shown by Lu et al. (2017), which requires that the gas heating ultimately derives the energy from the same SF process. The SNe heating scenario would naturally fit this requirement.

\subsubsection{Bar-Induced Shock Heating Scenario}

Fig. 12 displays the integrated CO (6-5) line emission contours overlaid on the HST F606W $(0.606 \mu \mathrm{m}) \mathrm{im}-$ age (Malkan et al. 1998) and HST F160W $(1.60 \mu \mathrm{m})$ image (Alonso-Herrero et al. 2006), respectively. The $H S T$ images aligned with our $\mathrm{CO}(6-5)$ data by matching our adopted AGN position with the brightest point in each optical image. As already mentioned before, the $\mathrm{CO}(6-5)$ emission has a good spatial correspondence with the dust lanes that can be seen in the optical and near-IR continuum images here. These roughly symmetrical dust lanes are induced by the inner stellar bar, both of which are more visible in a larger UV/optical image such as the one shown by Mulchaey \& Regan (1997). This correspondence between the $\mathrm{CO}(6-5)$ emission and the bar-induced dust lanes in NGC 5135 is similar to that observed in NGC 7130, another LIRG with a strong stellar bar (Zhao et al. 2016). Indeed NGC 5135 and NGC 7130 have many similarities: both are LIRGs with a strong circumnuclear star formation and a Seyfert $2 \mathrm{nu}-$ cleus. Circumnuclear dust lanes have been found in many spiral galaxies, though strong 2-arm dust lanes are found only in barred galaxies such as NGC 5135 and NGC 7130 (Martini et al. 2003).

According to the model of Athanassoula (1992), the 2-arm dust lanes are associated with the shock fronts triggered by the presence of a bar in a rotating gas disk. Thus, bar-induced shocks could be possible in the nuclear region of NGC 5135. Inside the dust lanes, the gas (and dust) density is significantly enhanced, but SF is suppressed by strong shears (Athanassoula 1992). This seems to imply that the warm dense gas traced by $\mathrm{CO}(6-5)$ and the SF regions traced by $\mathrm{Pa}-\alpha$ are not related to each other.

In order to account for the tight correlation between the $\mathrm{CO}(6-5)$ emission and the total dust emission on galaxy scale for LIRGs, one has to relate the $\mathrm{CO}(6-5)$ emitting gas to the SF activity in this scenario. It is still possible that the warm dense gas and the SF regions are related to each other, albeit their positions are slightly offset. It is known that some galaxies with weak stellar bars (therefore weaker shears in dust lanes) have SF in their dust lanes (Comte \& Duquennoy 1982; Martini et al. 2003). Hypothetically, one can envisage the following scenario: First, SF does occur in clouds of dense gas formed in the post-shock gas down stream from the barinduced shock front (the dust lane). Then these dense gas clouds will be rapidly consumed/destroyed by the $\mathrm{SF}$ and the associated feedback. In this scenario, under the assumption that the destruction time scale of the dense clouds is much shorter than the SF time scale associated with the Pa- $\alpha$ emission (a few Myrs), the spatial offset is the product of the SF time scale times the downstream velocity of the post-shock gas, which is a few $10 \mathrm{sm} \mathrm{s}^{-1}$ (Athanassoula 1992). This indeed results in an estimate for the offset of $\lesssim 100 \mathrm{pc}$. It is worth noting that similar offset between HiI regions and dust lanes associated with spiral arms in grand-design galaxies such as M 51 have been found in the literature, and Scoville et al. (2001) argued that it implies that the Hir regions develop subsequent to the time of maximum concentration of the dust and molecular clouds.

However, it is unclear how this bar-induced shock heating scenario for the $\mathrm{CO}(6-5)$ emission can be made to explain the similar variation in the $\mathrm{CO}(6-5)$-to-continuum flux ratio seen in IC 5179 (Zhao et al. 2017), which does not have a strong stellar bar.

\section{SUMMARY}

In this paper we present the results from our ALMA observations of the $\mathrm{CO}(6-5)$ line and its underlying dust continuum at $435 \mu \mathrm{m}$ in the nuclear region of the nearby LIRG, Seyfert 2 galaxy NGC 5135, at a physical resolution of $48 \times 40 \mathrm{pc}$. Our main findings are:

(1) The central AGN is undetected in either the 435 $\mu \mathrm{m}$ dust continuum or $\mathrm{CO}(6-5)$ line emission if its line velocity width is no less than $\sim 40 \mathrm{~km} \mathrm{~s}^{-1}$, resulting in an AGN contributes at most $1 \%$ of the integrated circumnuclear CO (6-5) flux seen in our ALMA observation. On the other hand, the non-detection in continuum emission may simply reflect the lack of sensitivity in our observation.

(2) The circum-nuclear CO (6-5) emission is resolved into gas clumps of radii of $45-180 \mathrm{pc}$ and line velocity widths of $60-88 \mathrm{~km} \mathrm{~s}^{-1}$. While the clump sizes are only slightly larger than typical giant molecular clouds in nearby spiral galaxies, their velocity widths are significantly higher. They fall into two categories: (i) The 5 clumps that are near some current star formation activity 
are likely to be in Virial equilibrium, and (ii) the other 2 clumps without clear current star formation activity nearby seem to be unbound unless there is significant external pressure.

(3) The clumps in Category (ii) have much higher $\mathrm{CO}(6-5)$ to dust continuum ratios than those in Category (i). Furthermore, the CO (6-5)-to-continuum ratios show an increasing trend with the [Fe II]-to-Br- $\gamma$ ratios, which we interpret as evidence for supernova-driven shocked gas providing a significant contribution to the $\mathrm{CO}(6-5)$ emission.

(4) The clumps are distributed along the symmetric optical dust lanes associated with the stellar bar at the center of the galaxy. Like NGC 7130, another barred Seyfert galaxy, the gas concentrations could be a result of the bar-induced instability and is subject to bar-induced shock heating.

\section{ACKNOWLEDGMENTS}

We thank an anonymous referee for a number of very constructive comments. We thank Drs. Cheng Cheng,
Luis Colina, Adam Leroy, Claudio Ricci, and Chentao Yang for their insightful comments and/or useful communications during the preparation of the manuscript. This paper makes use of the following ALMA data: ADS/JAO.ALMA\#2013.1.00524.S. ALMA is a partnership of ESO (representing its member states), NSF (USA), and NINS (Japan), together with NRC (Canada) and NSC and ASIAA (Taiwan), in cooperation with the Republic of Chile. The Joint ALMA Observatory is operated by ESO, AUI/NRAO, and NAOJ. This work is supported in part by the National Key R\&D Program of China grant \#2017YFA0402704, the NSFC grant \#11673028 and \#11673057, and by the Chinese Academy of Sciences (CAS), through a grant to the CAS South America Center for Astronomy (CASSACA) in Santiago, Chile. C.C. acknowledges support by NSFC grant No.11503013. Y.G. acknowledges support by NSFC grants No.11173059, 11390373, and 11420101002. H.W. acknowledges support by NSFC grant No.11733006. V.K. acknowledges support from the FONDECYT grant No.3160117. T.D.-S. acknowledges support from ALMA-CONICYT project 31130005 and FONDECYT regular project 1151239.

\section{REFERENCES}

Aalto, S., Muller, S., Costagliola, F., et al. 2017, A\&A, 608, A22

Alonso-Herrero, A., Rieke, G. H., Rieke, M. J., et al. 2006, ApJ, 650,835

Alonso-Herrero, A., Rieke, M. J., Rieke, G. H., \& Ruiz, M. 1997, ApJ, 482, 747

Armus, L., Mazzarella, J. M., Evans, A. S., et al. 2009, PASP, 121,559

Asmus, D., Gandhi, P., Hönig, S. F., Smette, A., \& Duschl, W. J. 2015, MNRAS, 454, 766

Athanassoula, E. 1992, MNRAS, 259, 345

Bedregal, A. G., Colina, L., Alonso-Herrero, A., \& Arribas, S. 2009, ApJ, 698, 1852

Bradford, C. M., Nikola, T., Stacey, G. J., et al. 2003, ApJ, 586, 891

Bryant, P. M., \& Scoville, N. Z. 1999, AJ, 117, 2632

Caputi, K. I., Lagache, G., Yan, L., et al. 2007, ApJ, 660, 97

Colina, L., Pereira-Santaella, M., Alonso-Herrero, A., Bedregal, A. G., \& Arribas, S. 2012, ApJ, 749, 116

Comte, G., \& Duquennoy, A. 1982, A\&A, 114, 7

Díaz-Santos, T., Alonso-Herrero, A., Colina, L., et al. 2008, ApJ, 685,211

Díaz-Santos, T., Armus, L., Charmandaris, V., et al. 2017, ApJ, 846, 32

Downes, D., \& Solomon, P. M. 1998, ApJ, 507, 615

Espada, D., Matsushita, S., Miura, R. E., et al. 2017, ApJ, 843, 136

Evans, A. S., Mazzarella, J. M., Surace, J. A., \& Sanders, D. B. 2002, ApJ, 580, 749

Falcón-Barroso, J., Ramos Almeida, C., Böker, T., et al. 2014, MNRAS, 438, 329

Field, G. B., Blackman, E. G., \& Keto, E. R. 2011, MNRAS, 416, 710

Fukazawa, Y., Hiragi, K., Mizuno, M., et al. 2011, ApJ, 727, 19

Gao, Y., \& Solomon, P. M. 1999, ApJ, 512, L99

García-Burillo, S., Combes, F., Usero, A., et al. 2014, A\&A, 567, A125

García-Burillo, S., Combes, F., Ramos Almeida, C., et al. 2016, ApJ, 823, L12

González Delgado, R. M., Heckman, T., Leitherer, C., et al. 1998, ApJ, 505, 174

Greenhouse, M. A., Woodward, C. E., Thronson, Jr., H. A., et al. 1991, ApJ, 383, 164

Griffin, M. J., Abergel, A., Abreu, A., et al. 2010, A\&A, 518, L3

Gruppioni, C., Pozzi, F., Rodighiero, G., et al. 2013, MNRAS, 432,23
Heyer, M., Krawczyk, C., Duval, J., \& Jackson, J. M. 2009, ApJ, 699, 1092

Kamenetzky, J., Glenn, J., Rangwala, N., et al. 2012, ApJ, 753, 70

Kawamura, A., Mizuno, Y., Minamidani, T., et al. 2009, ApJS, 184,1

Larson, R. B. 1981, MNRAS, 194, 809

Le Floc'h, E., Papovich, C., Dole, H., et al. 2005, ApJ, 632, 169

Leroy, A. K., Bolatto, A. D., Ostriker, E. C., et al. 2015, ApJ, 801,25

Levenson, N. A., Weaver, K. A., Heckman, T. M., Awaki, H., \& Terashima, Y. 2004, ApJ, 602, 135

Lu, N., Zhao, Y., Xu, C. K., et al. 2014, ApJ, 787, L23

Lu, N., Zhao, Y., Díaz-Santos, T., et al. 2017, ApJS, 230, 1

Magnelli, B., Elbaz, D., Chary, R. R., et al. 2009, A\&A, 496, 57

—. 2011, A\&A, 528, A35

Malkan, M. A., Gorjian, V., \& Tam, R. 1998, ApJS, 117, 25

Martini, P., Regan, M. W., Mulchaey, J. S., \& Pogge, R. W. 2003, ApJS, 146, 353

McMullin, J. P., Waters, B., Schiebel, D., Young, W., \& Golap, K. 2007, in Astronomical Society of the Pacific Conference Series, Vol. 376, Astronomical Data Analysis Software and Systems XVI, ed. R. A. Shaw, F. Hill, \& D. J. Bell, 127

Meijerink, R., Kristensen, L. E., Weiß, A., et al. 2013, ApJ, 762, L16

Moorwood, A. F. M., \& Oliva, E. 1988, A\&A, 203, 278

Mouri, H., Kawara, K., \& Taniguchi, Y. 1993, ApJ, 406, 52

Mouri, H., Nishida, M., Taniguchi, Y., \& Kawara, K. 1990, ApJ, 360,55

Muñoz Marín, V. M., González Delgado, R. M., Schmitt, H. R., et al. 2007, AJ, 134, 648

Mulchaey, J. S., \& Regan, M. W. 1997, ApJ, 482, L135

Mullaney, J. R., Alexander, D. M., Goulding, A. D., \& Hickox, R. C. 2011, MNRAS, 414, 1082

Nikola, T., Stacey, G. J., Brisbin, D., et al. 2011, ApJ, 742, 88

Oka, T., Hasegawa, T., Sato, F., et al. 2001, ApJ, 562, 348

Pellegrini, E. W., Smith, J. D., Wolfire, M. G., et al. 2013, ApJ, 779, L19

Phillips, M. M., Charles, P. A., \& Baldwin, J. A. 1983, ApJ, 266, 485

Pilbratt, G. L., Riedinger, J. R., Passvogel, T., et al. 2010, A\&A, 518, L1

Ramos Almeida, C., Pérez García, A. M., \& Acosta-Pulido, J. A. 2009, ApJ, 694, 1379

Ramos Almeida, C., Pérez García, A. M., Acosta-Pulido, J. A., et al. 2006, ApJ, 645, 148 
Rangwala, N., Maloney, P. R., Wilson, C. D., et al. 2015, ApJ, 806,17

Rangwala, N., Maloney, P. R., Glenn, J., et al. 2011, ApJ, 743, 94

Riffel, R., Rodríguez-Ardila, A., Aleman, I., et al. 2013, MNRAS, 430, 2002

Rigopoulou, D., Hurley, P. D., Swinyard, B. M., et al. 2013, MNRAS, 434, 2051

Rodríguez-Ardila, A., Pastoriza, M. G., Viegas, S., Sigut, T. A. A., \& Pradhan, A. K. 2004, A\&A, 425, 457

Rodríguez-Ardila, A., Riffel, R., \& Pastoriza, M. G. 2005, MNRAS, 364, 1041

Rosenberg, M. J. F., Meijerink, R., Israel, F. P., et al. 2014, A\&A, 568, A90

Rosolowsky, E., \& Blitz, L. 2005, ApJ, 623, 826

Sakamoto, K., Mao, R.-Q., Matsushita, S., et al. 2011, ApJ, 735, 19

Sanders, D. B., \& Mirafbel, I. F. 1996, ARA\&A, 34, 749

Sanders, D. B., Scoville, N. Z., \& Soifer, B. T. 1991, ApJ, 370, 158

Sanders, D. B., Scoville, N. Z., Young, J. S., et al. 1986, ApJ, 305, L45

Scoville, N., Sheth, K., Aussel, H., et al. 2016, ApJ, 820, 83

Scoville, N. Z., Polletta, M., Ewald, S., et al. 2001, AJ, 122, 3017

Singh, V., Risaliti, G., Braito, V., \& Shastri, P. 2012, MNRAS, 419, 2089

Sliwa, K., Wilson, C. D., Aalto, S., \& Privon, G. C. 2017, ApJ, 840, L11
Soifer, B. T., Sanders, D. B., Madore, B. F., et al. 1987, ApJ, 320,238

Solomon, P. M., Downes, D., Radford, S. J. E., \& Barrett, J. W. 1997, ApJ, 478, 144

Solomon, P. M., Rivolo, A. R., Barrett, J., \& Yahil, A. 1987, ApJ, 319,730

Solomon, P. M., \& Sage, L. J. 1988, ApJ, 334, 613

Spaans, M., \& Meijerink, R. 2008, ApJ, 678, L5

Spinoglio, L., Pereira-Santaella, M., Busquet, G., et al. 2012, ApJ, 758, 108

Turner, T. J., George, I. M., Nandra, K., \& Mushotzky, R. F. 1997, ApJS, 113, 23

Ulvestad, J. S., \& Wilson, A. S. 1989, ApJ, 343, 659

Wang, J. L., Xia, X. Y., Mao, S., et al. 2006, ApJ, 649, 722

Williams, J. P., de Geus, E. J., \& Blitz, L. 1994, ApJ, 428, 693

Wilson, C. D., Rangwala, N., Glenn, J., et al. 2014, ApJ, 789, L36

Wootten, A., \& Thompson, A. R. 2009, IEEE Proceedings, 97, 1463

Xu, C. K., Cao, C., Lu, N., et al. 2014, ApJ, 787, 48

-. 2015, ApJ, 799, 11

Zhao, Y., Lu, N., Xu, C. K., et al. 2016, ApJ, 820, 118

Zhao, Y., Lu, N., Díaz-Santos, T., et al. 2017, ApJ, 845, 58 

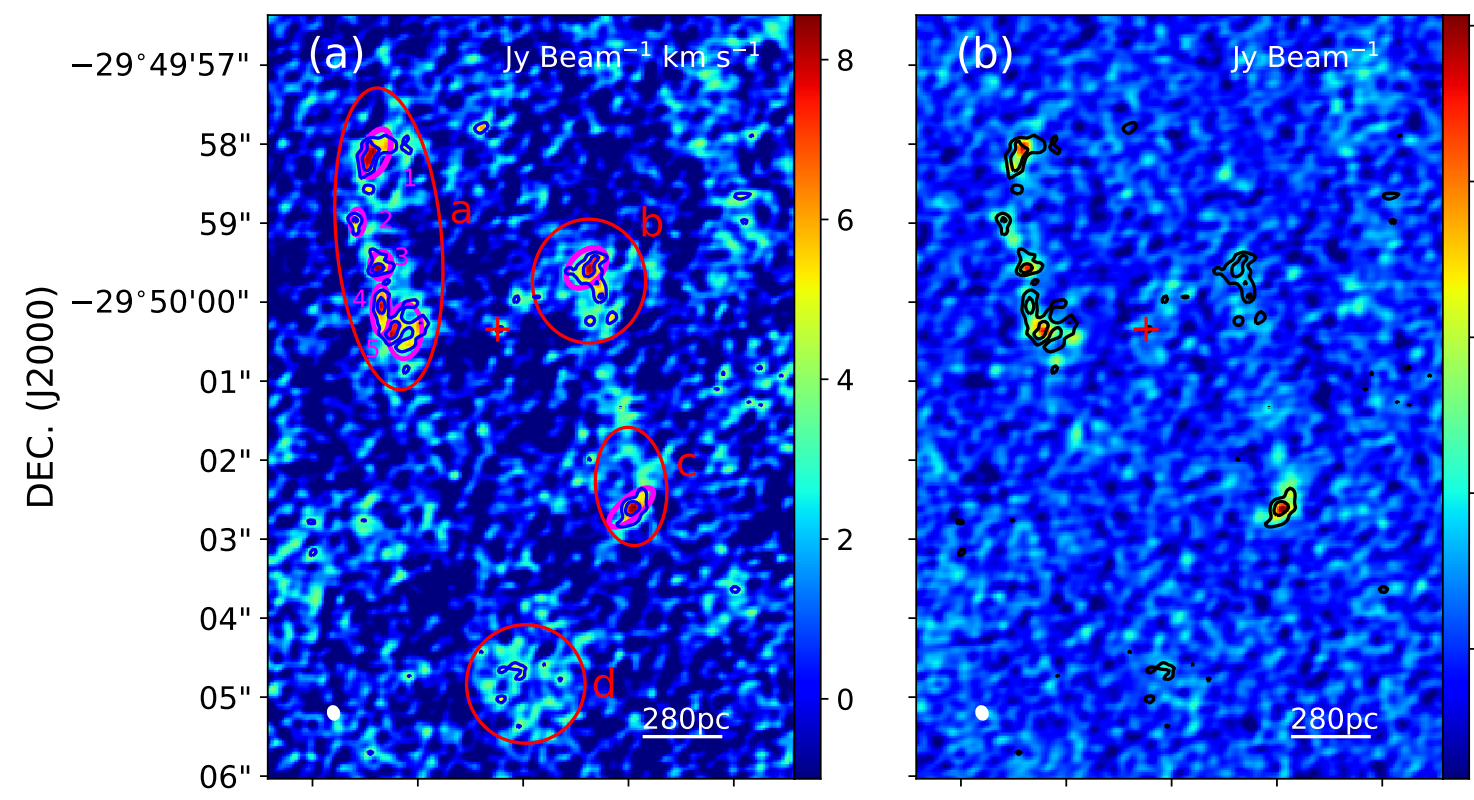

0.024

0.016

0.012

0.008
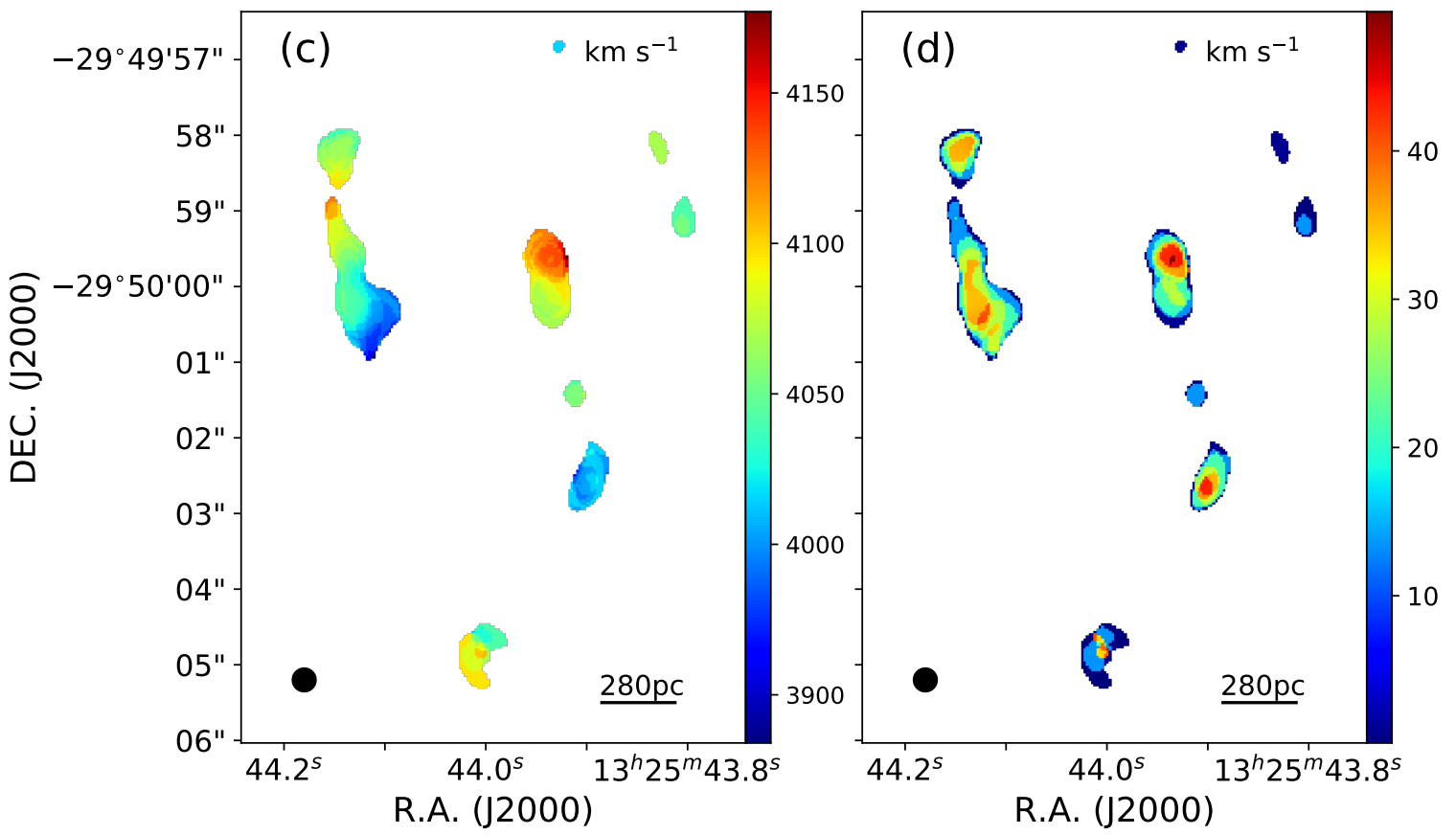

Fig. 1. - Panels (a) and (b): Contours of the frequency-integrated CO (6-5) intensity overlaid on the images of the same integrated $\mathrm{CO}$ (6-5) intensity in (a) and the $435 \mu \mathrm{m}$ dust continuum emission in (b). Panels (c) and (d) are respectively the line velocity field (moment 1 ) and the velocity dispersion (moment 2) maps of the $\mathrm{CO}(6-5)$ line emission obtained from the uv-taper image. The images in (c) and (d) are generated by using only those spaxels above $4-\sigma_{c h}$, where $\sigma_{c h}$ is the r.m.s noise per frequency channel $\left(\sigma_{c h}=40 \mathrm{mJy}\right.$ beam ${ }^{-1}$ for uv-taper image). The contours in panels (a) and (b) are shown at $[3,5] \times \sigma$ (where the noise $\sigma=1.2 \mathrm{Jy} \mathrm{beam}^{-1} \mathrm{kms}^{-1}$ ). The unit of the color bar in each panel is given near the upper-right color. The filed ellipse in white near the lower-left corner in (a) or (b) is the ALMA beam. The large ellipses in red in (a) mark the regions for spectrum and flux extractions given in Table 2. The red plus sign marks the AGN position adopted. The figures are before the primary beam correction integrated over the barycentric velocities from 3,971 to $4,157 \mathrm{~km} \mathrm{~s}^{-1}$. 


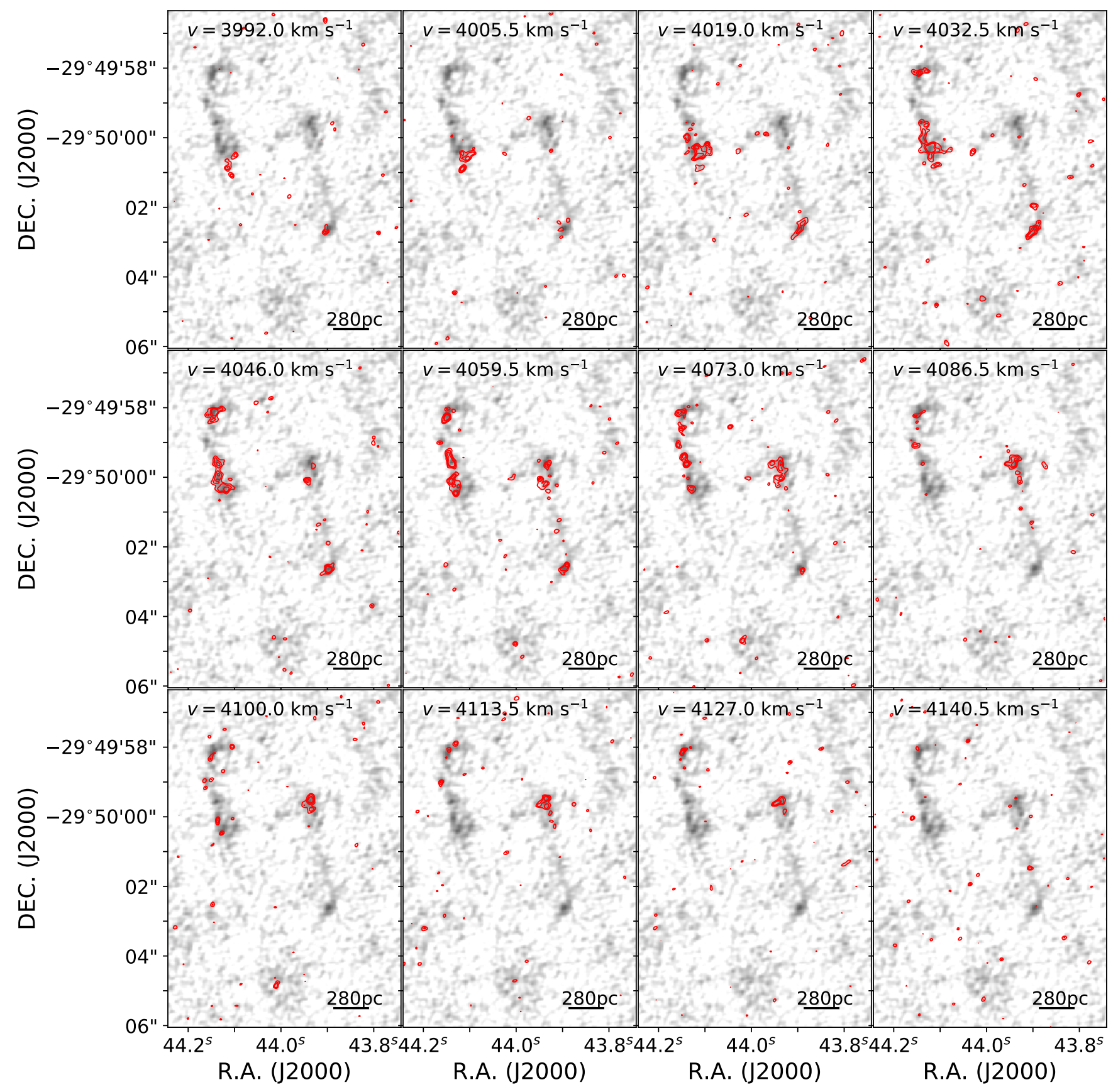

FIG. 2.- Channel maps of the $\mathrm{CO}(6-5)$ line emission (in contours), each overlaid on the image of the total, frequency-integrated CO (6-5) emission (e.g., from Fig. 1a). The channel interval is $13.5 \mathrm{~km} \mathrm{~s}^{-1}$, with the channel central (barycentric) velocity shown in each channel map. 

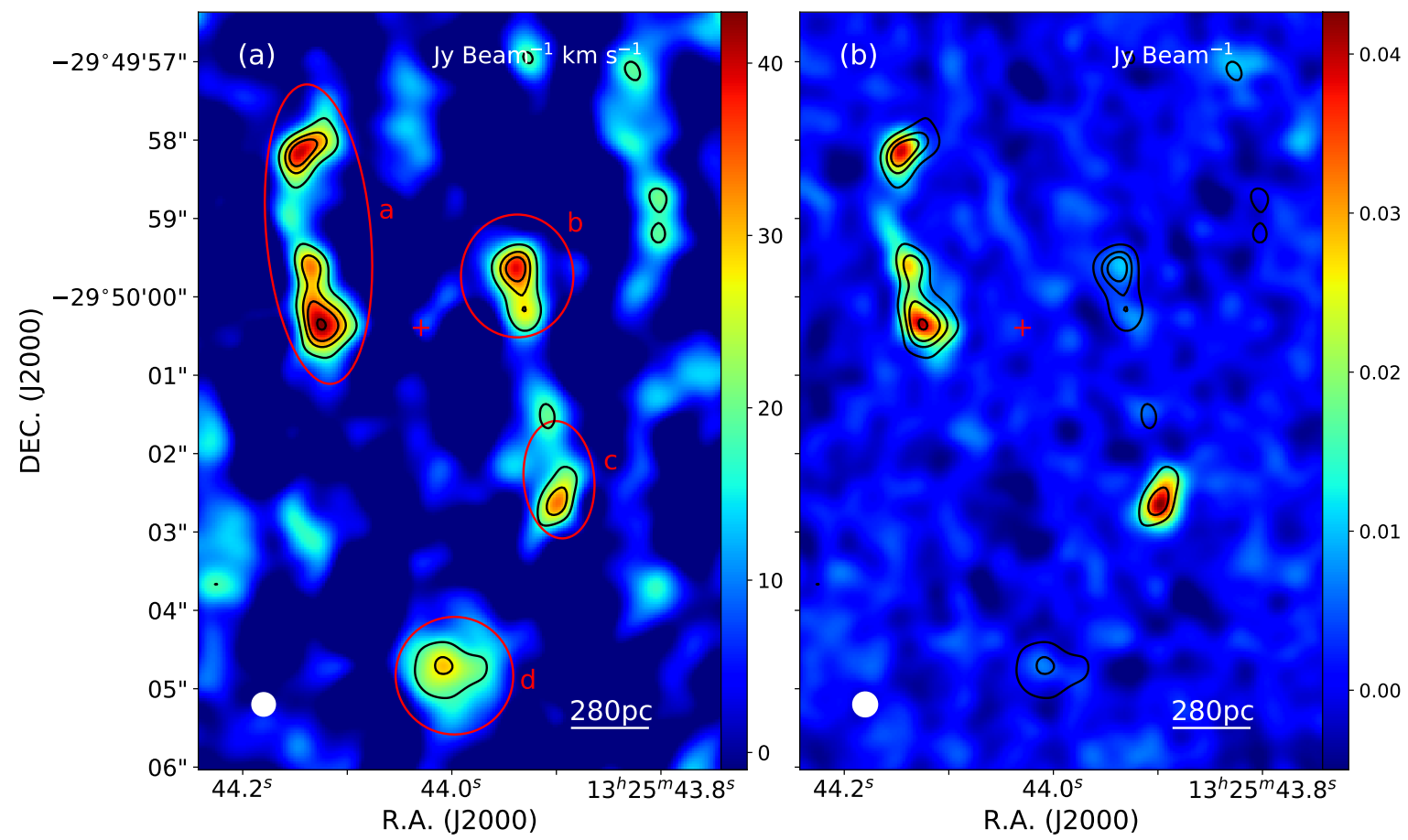

FIG. 3.- Same as the panels (a) and (b) in Fig. 1, respectively, but using the CO (6-5) and continuum data at a larger effective beam. The contour levels are $[3,4,5,6] \times \sigma\left(\sigma=8 \mathrm{Jy}_{\text {beam }}^{-1} \mathrm{~km} \mathrm{~s}^{-1}\right.$, with the beam size of $0.4^{\prime \prime} \times 0.4^{\prime \prime}$ here as shown by the filled ellipse in white in each panel.

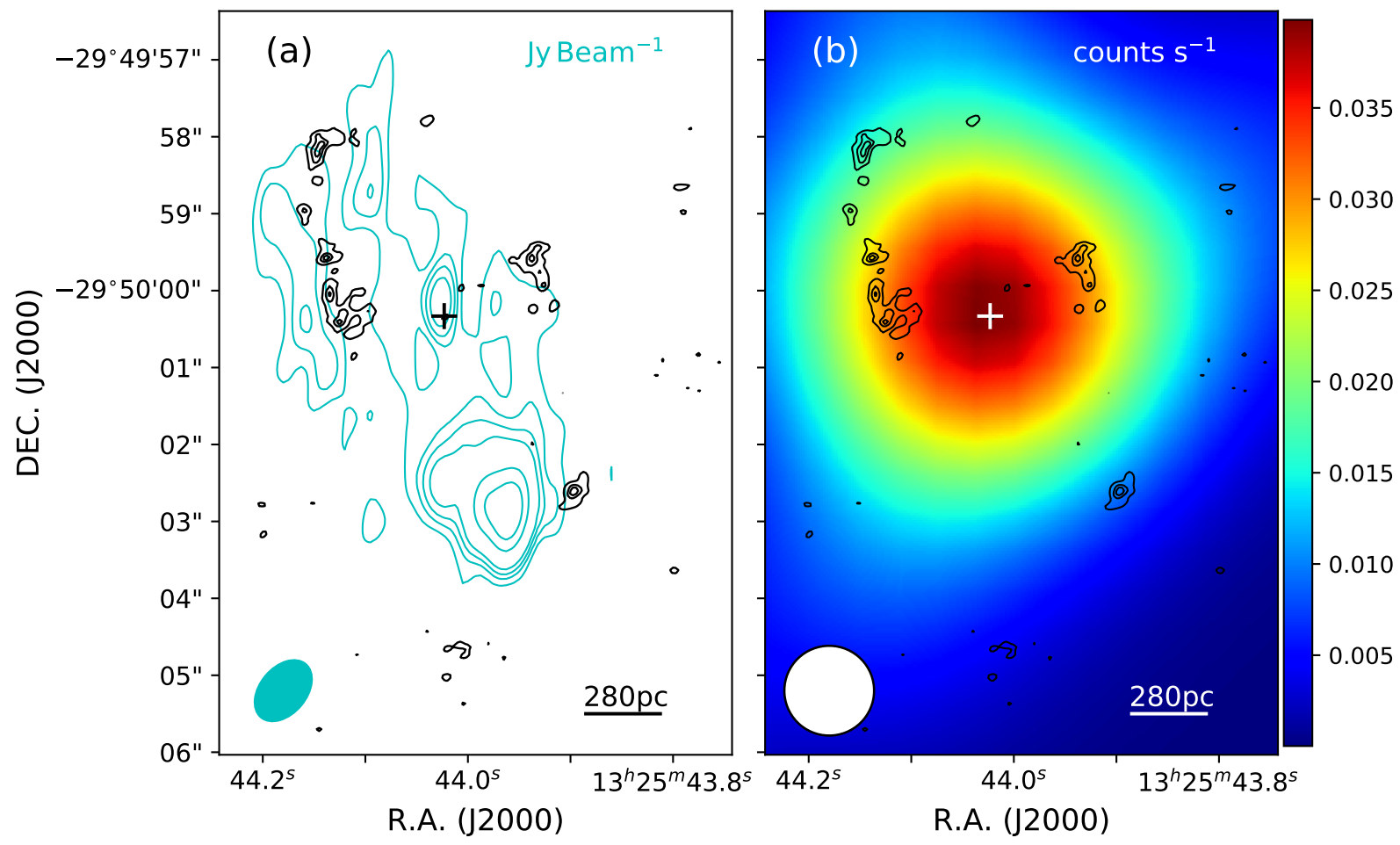

FIG. 4. - Black contours of the integrated $\mathrm{CO}(6-5)$ line emission overlaid on (a) an VLA radio cyan contours at $6 \mathrm{~cm}$ (the cyan contour levels are $[3,5,7,10,30,50] \times \sigma\left(\sigma=1.2 \mathrm{e}-04\right.$ Jybeam $\left.^{-1}\right)$ and (b) a Chandra $4-8 \mathrm{kev} \mathrm{X}$-ray image. The black contour level are $[3,5,6] \times \sigma$ $\left(\sigma=1.2\right.$ Jybeam $\left.^{-1} \mathrm{kms}^{-1}\right)$. The white plus sign in each panel presents the adopted AGN location. 

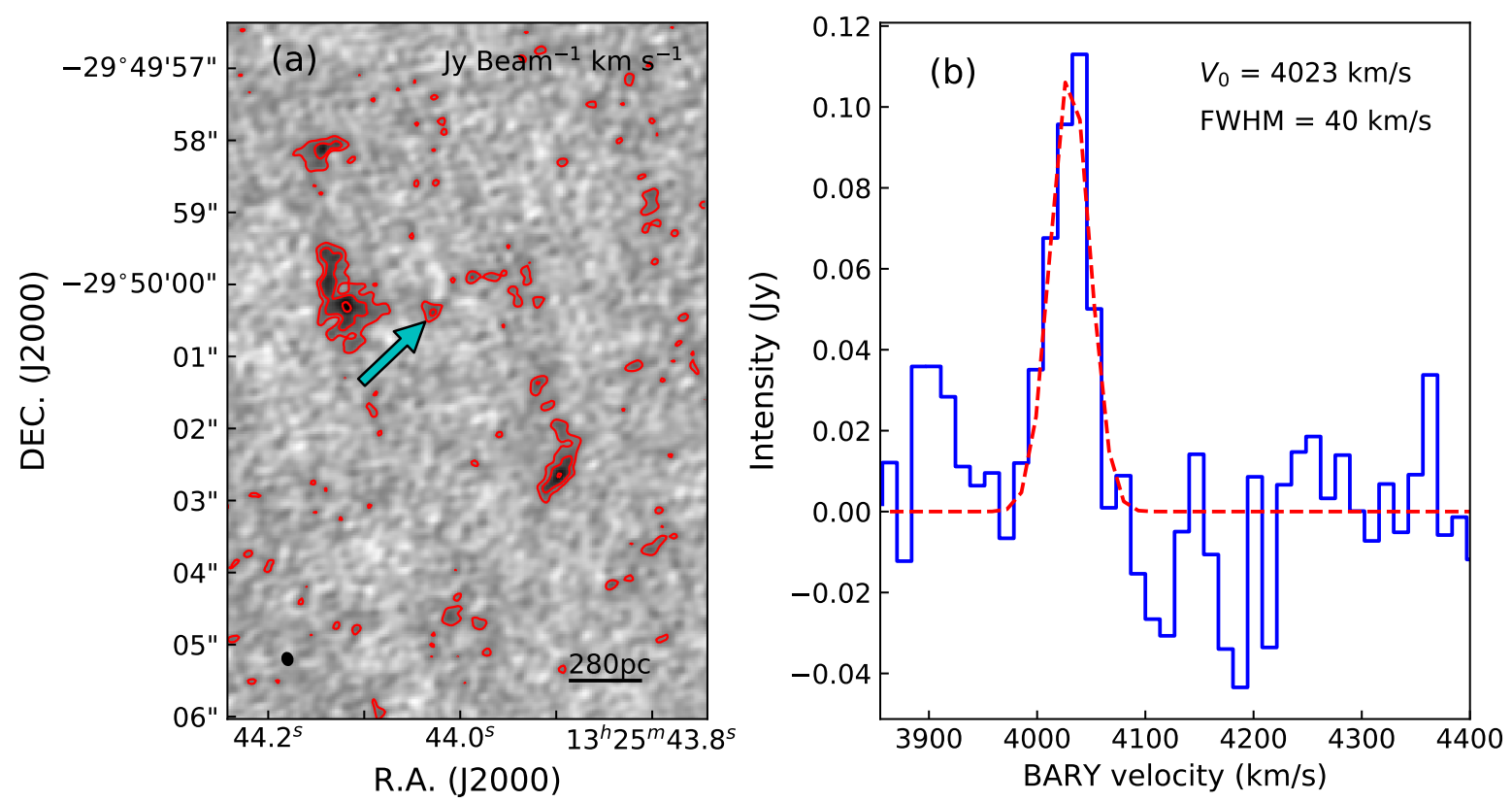

FIG. 5.- (a) Contours of the integrated CO (6-5) from 4019 to $4032 \mathrm{~km} / \mathrm{s}$ on the image of the same integrated CO (6-5) intensity. The cyan arrow points to the central AGN. The contour levels are $[3,5,8] \times \sigma\left(\sigma=0.5\right.$ Jybeam $\left.^{-1} \mathrm{kms}^{-1}\right)$. And (b) the CO (6-5) spectrum at the central AGN. The central velocity $\left(V_{0}\right)$ and FWHM of a Gaussian fit are given in the plot.

TABLE 1

BASIC PROPERTIES OF NGC 5135 AND ALMA OBSERVATION LOG

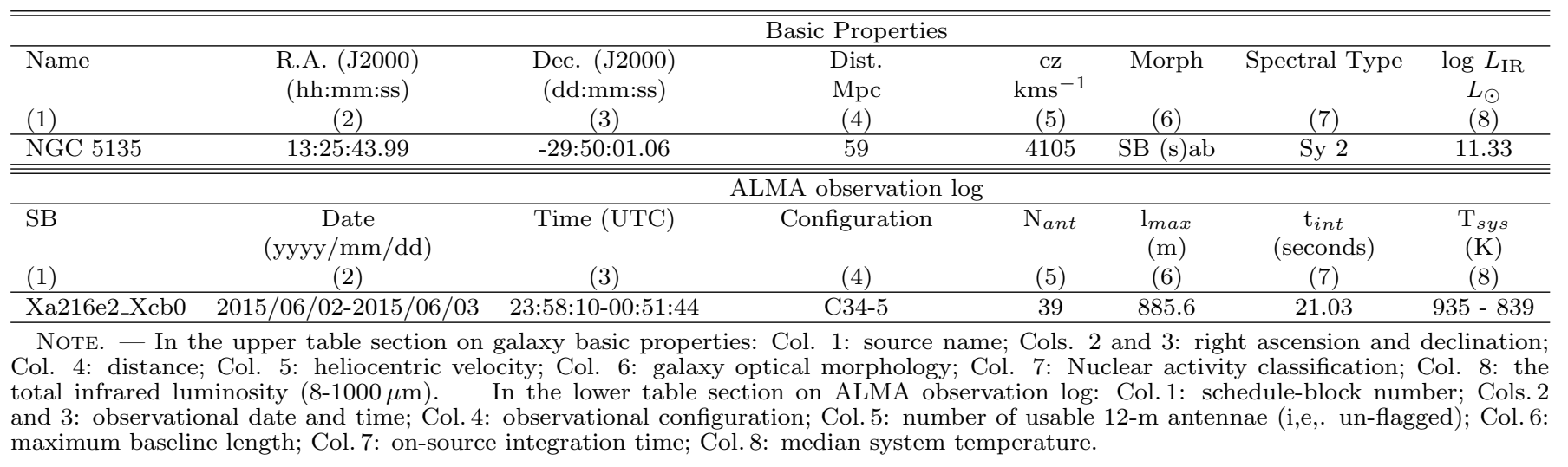




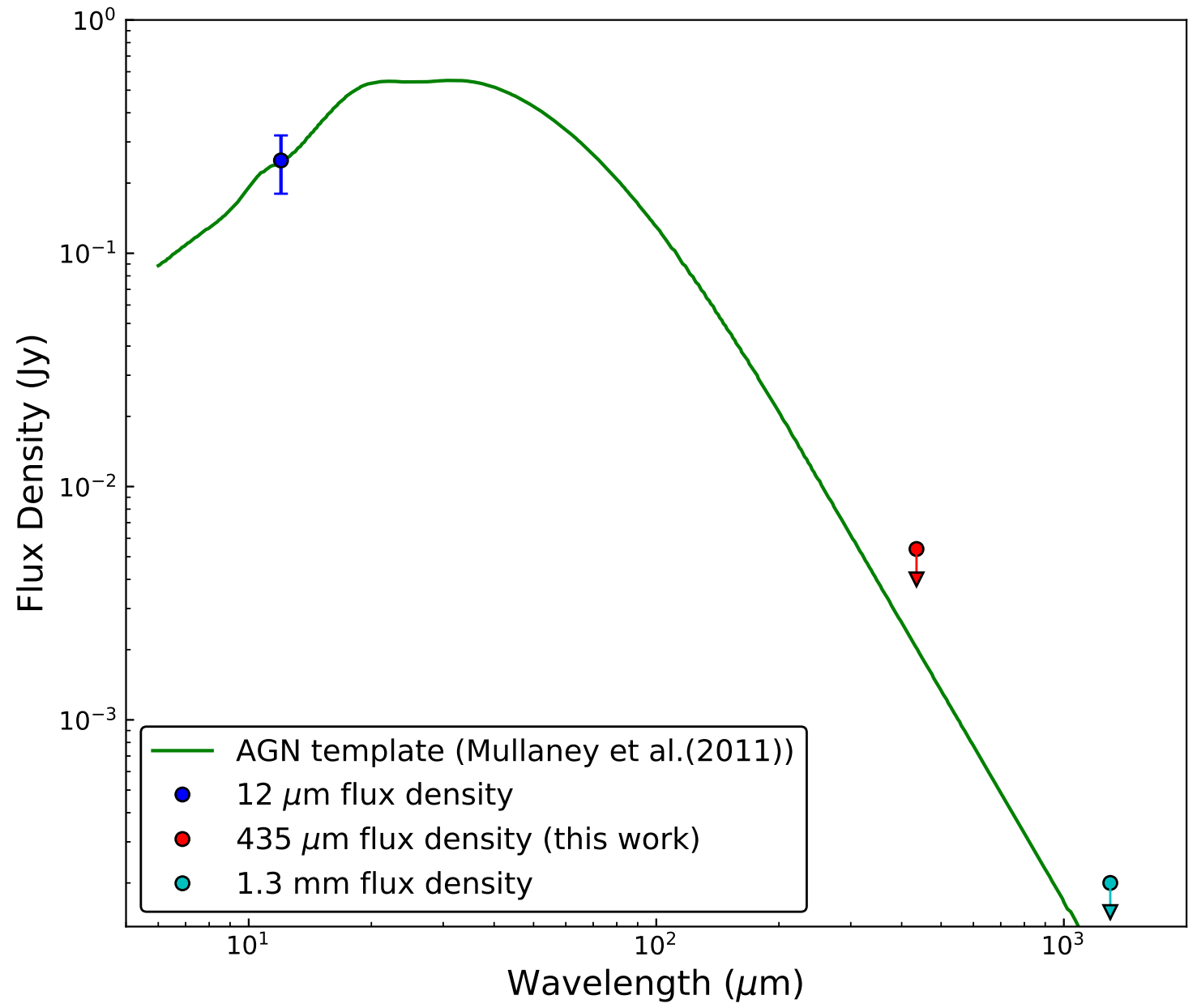

FIG. 6.- Plot of the empirical infrared spectrum (the green curve) of the AGN in NGC 5135, which is based the observed X-ray luminosity and anchored at the $12 \mu \mathrm{m}$ flux density measurement (the filled circle in blue). Also shown are two ALMA flux upper limits at $435 \mu \mathrm{m}$ and $1.3 \mathrm{~mm}$, respectively (see the text). 

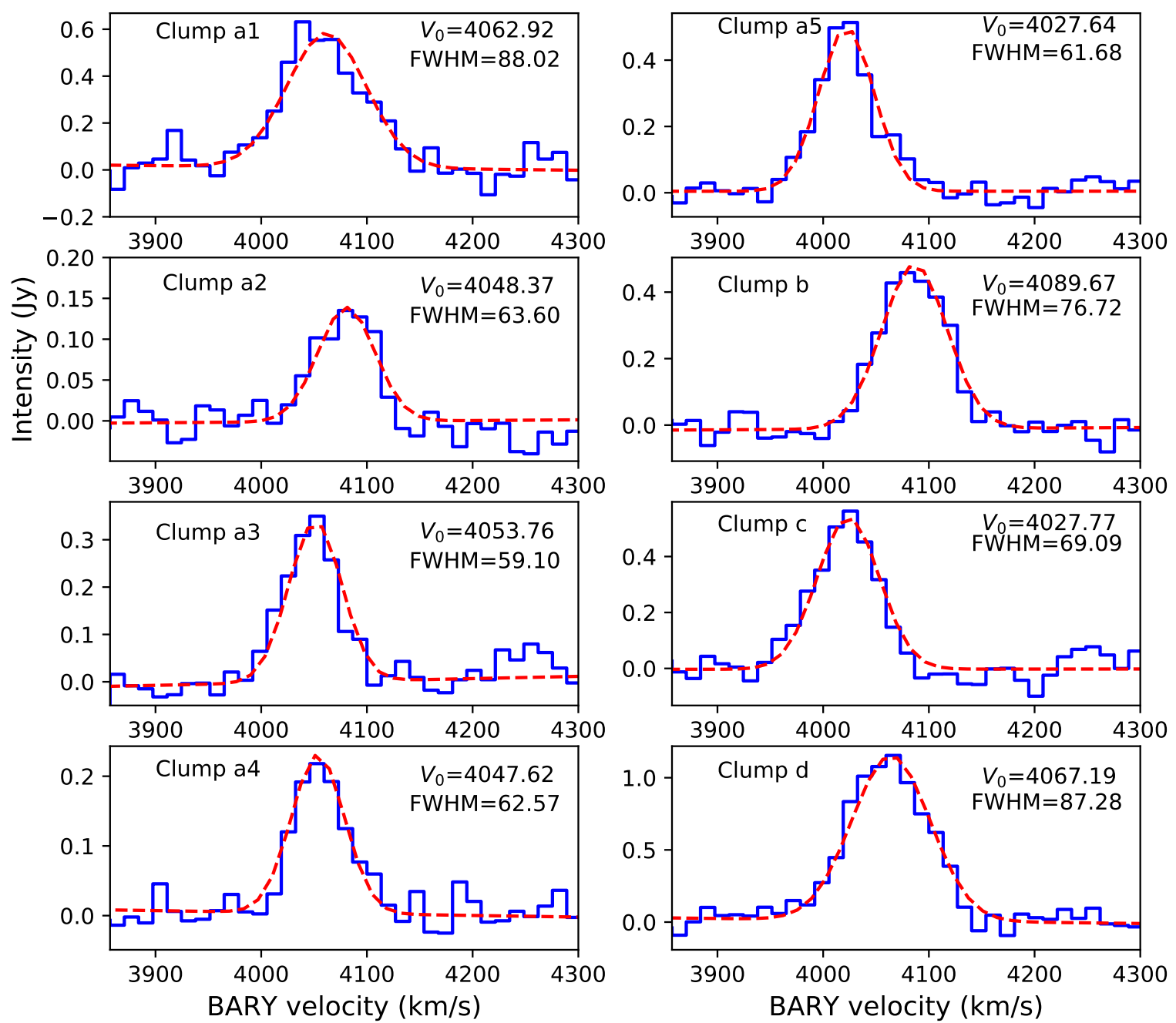

FIG. 7.- Spatially integrated CO (6-5) line profile of various clumps (a1, a2, a3, a4, a5, b, c, d). The central velocity ( $\left.V_{0}\right)$ and FWHM of a Gaussian fit are given in each plot. 


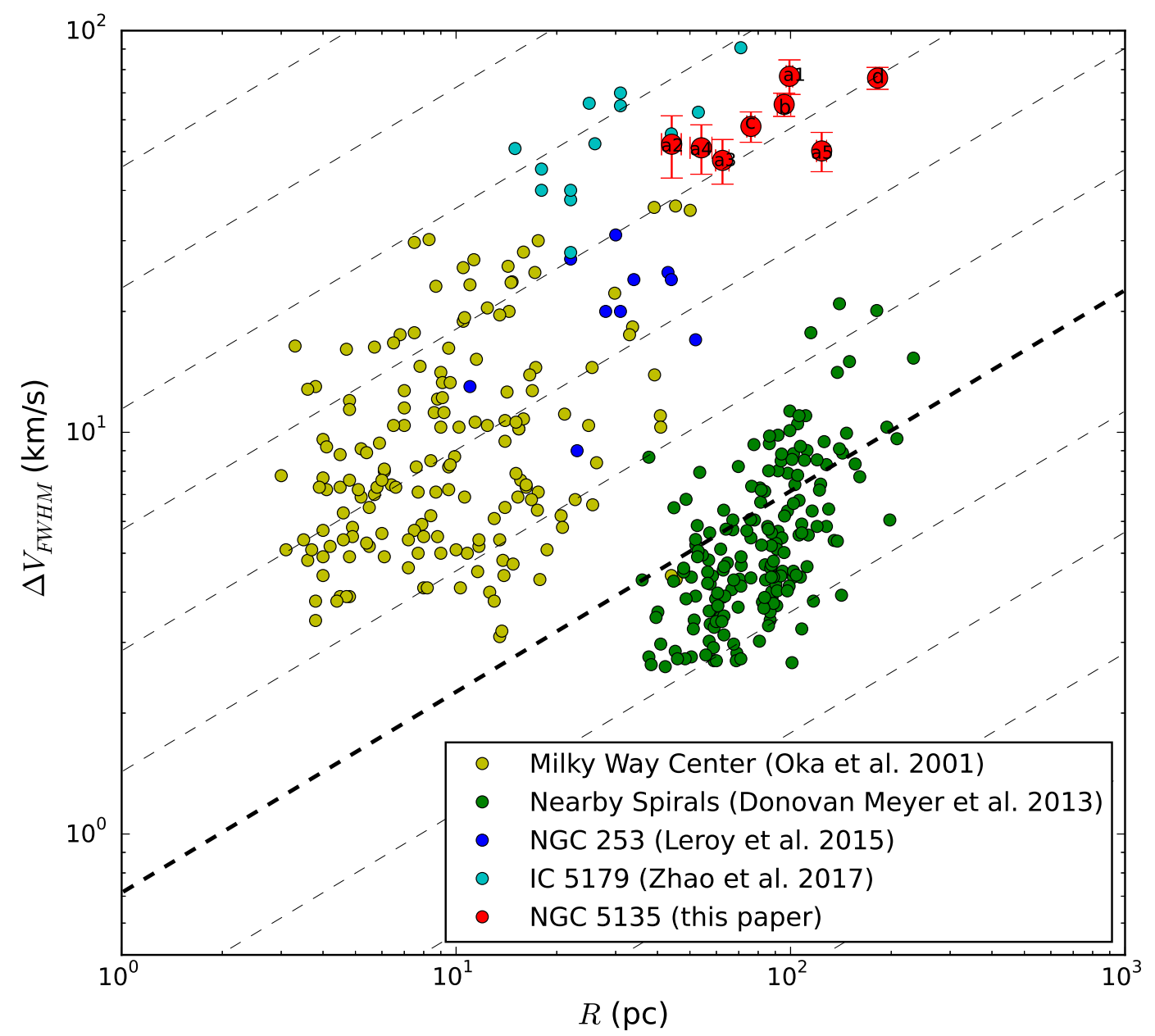

FIG. 8.- Plot of the CO (6-5) line width, $\Delta V_{\mathrm{FWHM}}$, as a function of the cloud radius $R$ for a sample of giant molecular clouds and gas complexes in the Milky Way and various local galaxies, adopted from Leroy et al. (2015). As a comparison, our NGC 5135 clumps are added (i.e., large filled circles labeled by the clump number). The light dashed lines follow $\Delta V_{\mathrm{FWHM}} \propto \mathrm{R}^{0.5}$, the relation expected for Virialized clouds with a fixed surface density $\Sigma$, spaced by a factor of 2 vertically. The thick dashed line is for $\Sigma \approx 285 \mathrm{M} \odot \mathrm{pc}^{-2}$. 


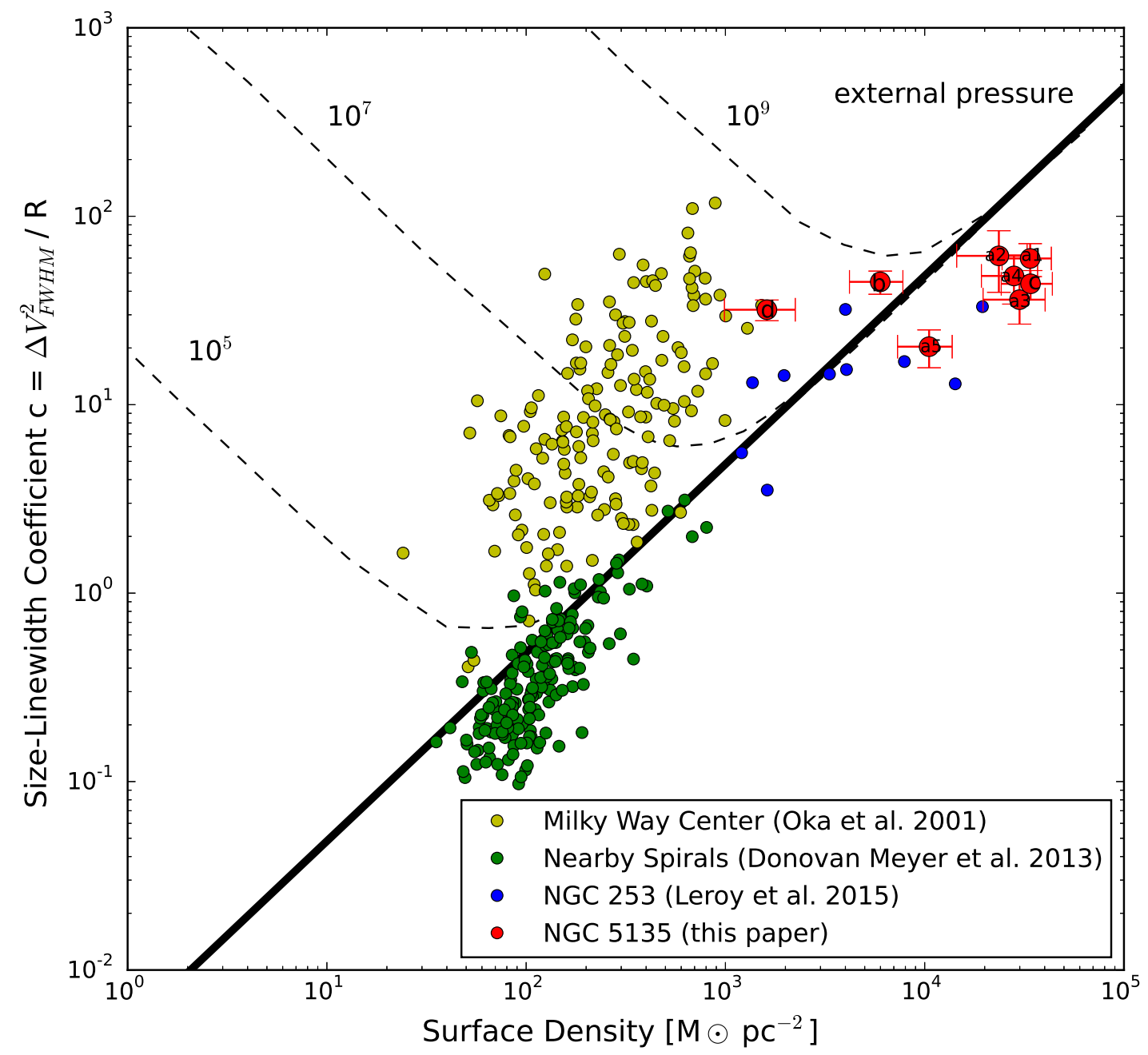

FIG. 9. - Plot of the cloud radius-line width coefficient, $\Delta V_{\mathrm{FWHM}}^{2} / R$, as a function of the gas surface density $\Sigma$, also adopted from Leroy et al. (2015), for the same data set as in Fig. 7. The gas clouds that are in Virial equilibrium (i.e., $M_{\text {mol }}=M_{\text {vir }}$ ) follow the thick line. Bound clouds with $M_{\mathrm{mol}}<M_{\mathrm{vir}}$ follow one of the thin curves representing various external pressures as labelled in terms of $\mathrm{P} / \mathrm{k}_{B}$ (in units of $\mathrm{cm}^{-3} \mathrm{~K}$ ). 

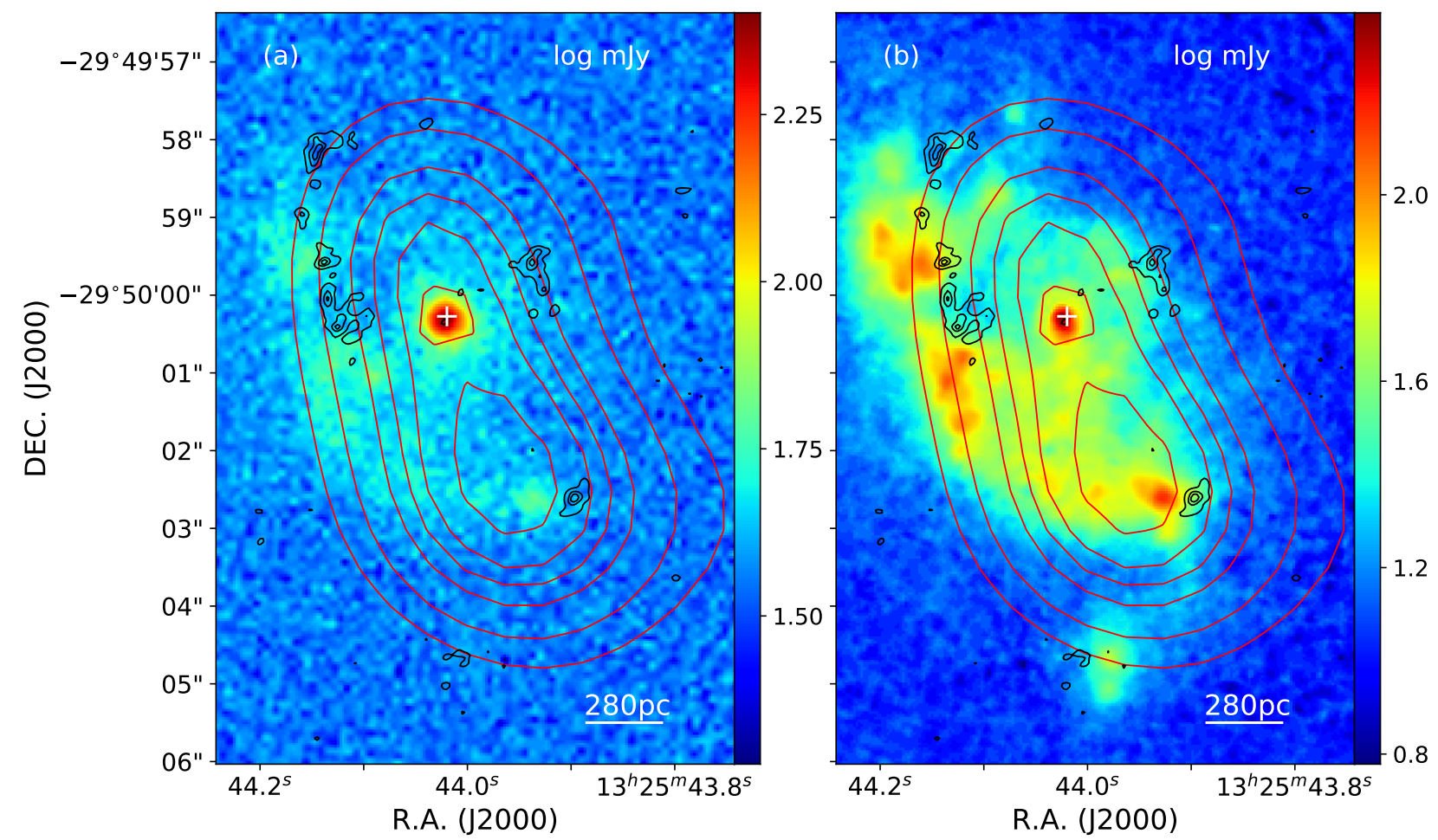

Fig. 10. - Integrated $\mathrm{CO}(6-5)$ line emission contours, at $[3,5,6] \times \sigma$ (where $\sigma=1.2 \mathrm{Jybeam}^{-1} \mathrm{kms}^{-1}$ ), overlaid on (a) a $8.7 \mu \mathrm{m}$ image dominated the PAH emission (in log scale) and (b) an image of the Pa- $\alpha$ line emission (in log scale). The red contours in each panel stands for the X-ray intensity of NGC 5135 (with the contours at 5, 20,30,40,70,150, 260 counts), obtained in the Chandra $0.4-8 \mathrm{keV}$ band by Levenson et al. (2004). The white plus sign in each panel marks the adopted AGN location. 


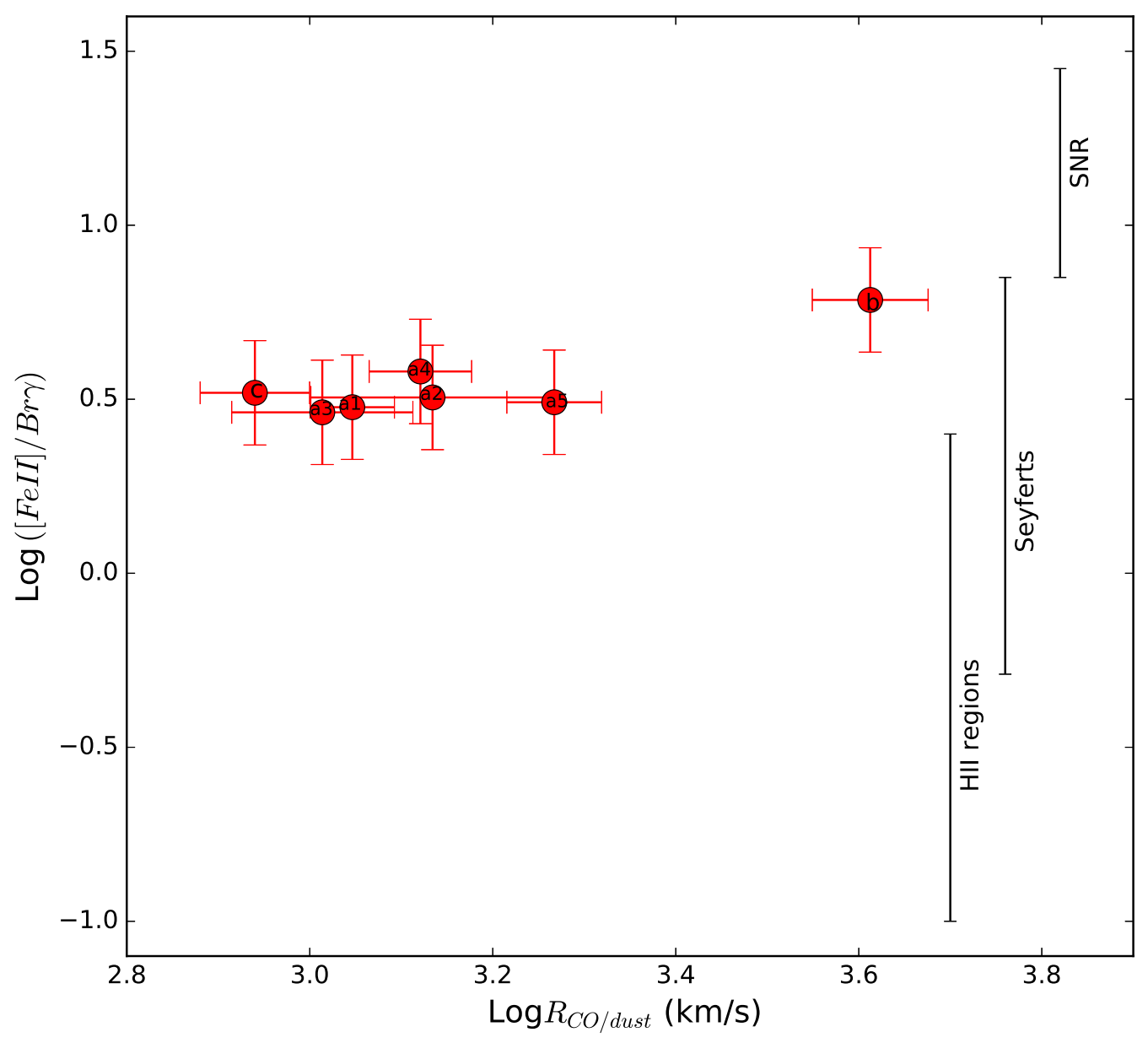

Fig. 11. - Plot of the [Fe II] $1.46 \mu \mathrm{m}$-to-Br- $\gamma$ line ratio as a function of the CO (6-5)-to-continuum flux ratio for the NGC 5135 clumps as labelled. The extinction corrected [Fe II] and Br- $\gamma$ line surface brightnesses at the local of a clump are estimated from Bedregal et al. (2009) and the typical error is 0.15 dex. The typical ratios for different types of astrophysical objects are noted in the plot (see the text). The clump $d$ is not plotted here as it does not have the corresponding [Fe II] or Br- $\gamma$ data. 

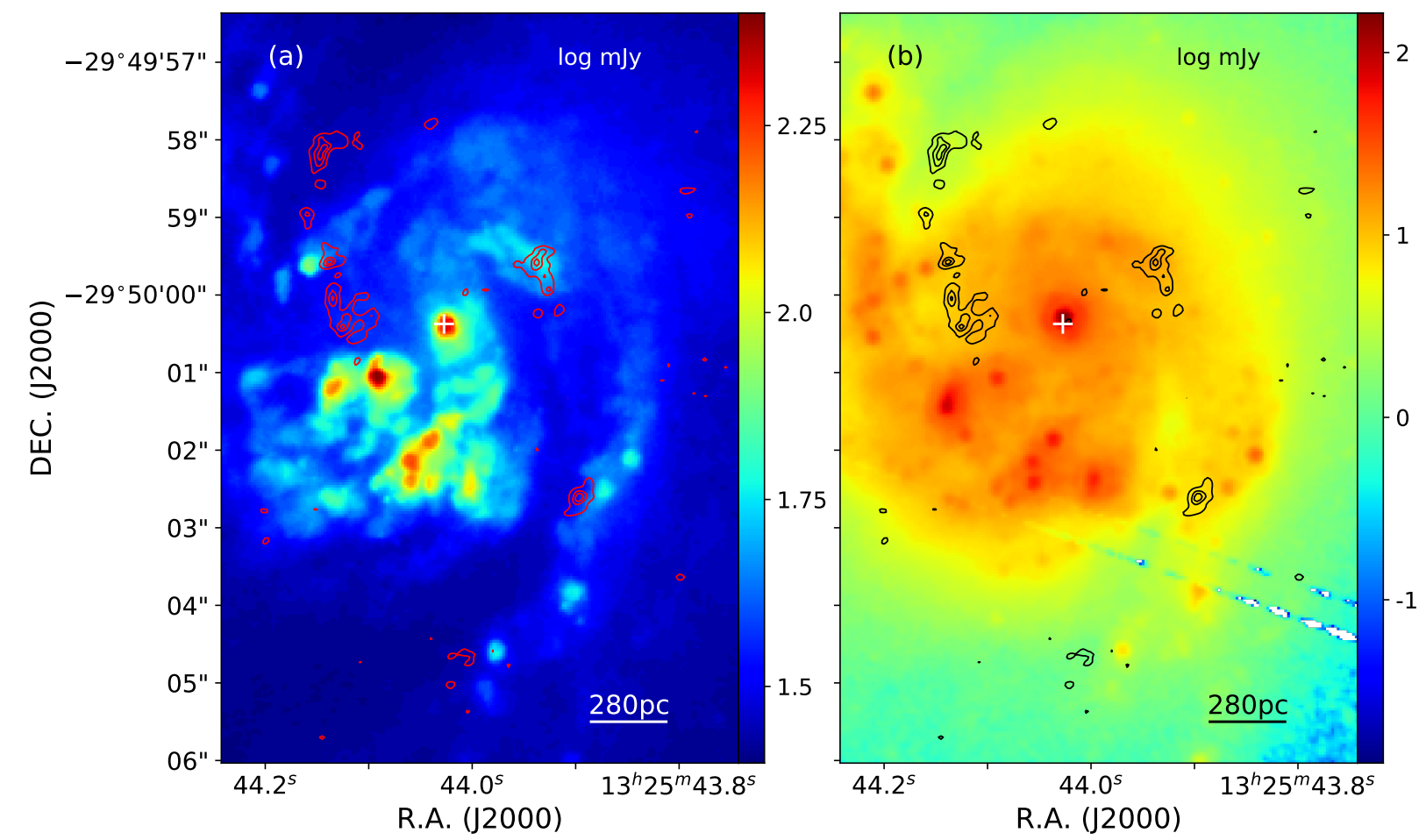

FIG. 12.- Integrated $\mathrm{CO}(6-5)$ line emission contours, at $[3,5,6] \times \sigma$ (where $\sigma=1.2 \mathrm{Jybeam}^{-1} \mathrm{kms}^{-1}$ ), overlaid on (a) an HST F606W $(0.606 \mu \mathrm{m}$, in log scale) image and (b) an HST F160W $(1.60 \mu \mathrm{m}$, in log scale) image. The white plus sign in each panel marks the AGN position. 
TABLE 2

Physical PROPERTIES OF THE INDIVIDUAL Clumps IN OUR CO (6-5) IMAGE

\begin{tabular}{|c|c|c|c|c|c|c|c|c|c|c|c|c|}
\hline No. & $\begin{array}{l}\text { Size } \\
"{ }^{\prime \prime}\end{array}$ & $\begin{array}{l}\text { PA } \\
\text { Deg }\end{array}$ & $\begin{array}{l}\text { Radius } \\
\text { pc }\end{array}$ & $\begin{array}{c}V_{0} \\
\mathrm{kms}^{-1} \\
(5)\end{array}$ & $\begin{array}{c}\Delta V_{\mathrm{FWHM}} \\
\mathrm{kms}^{-1}\end{array}$ & $\begin{array}{c}\mathrm{f}_{\text {peak }} \\
\mathrm{Jy} \mathrm{beam}^{-1} \\
\mathrm{~km} \mathrm{~s}^{-1} \\
(7)\end{array}$ & $\begin{array}{l}\mathrm{f}_{\mathrm{CO}(6-5)} \\
\mathrm{Jy} \mathrm{kms}^{-1}\end{array}$ & $\begin{array}{l}\mathrm{I}_{\text {cont }} \\
\mathrm{mJy}\end{array}$ & $\begin{array}{c}\mathrm{M}_{\mathrm{vir}} \\
\times 10^{7} \mathrm{M} \odot\end{array}$ & $\begin{array}{c}\mathrm{M}_{\mathrm{mol}} \\
\times 10^{8} \mathrm{M} \odot\end{array}$ & $\begin{array}{c}\mathrm{M}_{\mathrm{mol}}^{*} \\
\times 10^{8} \mathrm{M} \odot\end{array}$ & $\begin{array}{c}\mathrm{R}_{\mathrm{CO} / \text { cont }} \\
\mathrm{kms}^{-1}\end{array}$ \\
\hline a1 & $0.65 \times 0.34$ & 16.50 & 99.2 & 4062.9 & 88.0 & 13.3 & 91.1 & 81.8 & 37.0 & 10.5 & 12.6 & 1113.0 \\
\hline & $\pm 0.019 \times 0.010$ & & \pm 2.2 & \pm 3.0 & \pm 7.6 & & \pm 3.0 & \pm 8.4 & \pm 7.4 & \pm 2.9 & \pm 0.4 & \pm 119.0 \\
\hline a2 & $0.36 \times 0.10$ & -0.45 & 44.1 & 4084.4 & 63.6 & 9.2 & 15.5 & 11.4 & 7.6 & 1.4 & 2.1 & 1362.0 \\
\hline & $\pm 0.025 \times 0.012$ & & \pm 3.0 & \pm 3.7 & \pm 9.2 & & \pm 3.1 & \pm 2.7 & \pm 2.7 & \pm 0.5 & \pm 0.4 & \pm 419.0 \\
\hline a3 & $0.40 \times 0.25$ & 0.74 & 62.6 & 4053.7 & 59.1 & 10.6 & 29.6 & 28.7 & 8.9 & 3.7 & 4.1 & 1032.0 \\
\hline & $\pm 0.023 \times 0.014$ & & \pm 2.9 & \pm 4.0 & \pm 6.0 & & \pm 2.1 & \pm 6.2 & \pm 2.3 & \pm 1.2 & \pm 0.3 & \pm 236.0 \\
\hline $\mathrm{a} 4$ & $0.64 \times 0.19$ & 0.13 & 54.1 & 4047.6 & 62.6 & 9.6 & 28.5 & 21.6 & 9.4 & 2.6 & 3.9 & 1321.0 \\
\hline & $\pm 0.054 \times 0.011$ & & \pm 4.0 & \pm 2.4 & \pm 7.2 & & \pm 1.8 & \pm 2.4 & \pm 2.7 & \pm 0.7 & \pm 0.2 & \pm 171.0 \\
\hline a5 & $\begin{array}{c}0.72 \times 0.44 \\
\pm 0.023 \times 0.022\end{array}$ & 3.38 & $\begin{array}{l}123.9 \\
\pm 4.3\end{array}$ & $\begin{array}{c}4027.6 \\
\pm 1.2\end{array}$ & $\begin{array}{l}61.7 \\
\pm 5.6\end{array}$ & 9.6 & $\begin{array}{l}55.0 \\
\pm 1.5\end{array}$ & $\begin{array}{l}39.7 \\
\pm 6.3\end{array}$ & $\begin{array}{l}19.7 \\
\pm 4.5\end{array}$ & $\begin{array}{c}5.1 \\
\pm 1.5\end{array}$ & $\begin{array}{c}7.6 \\
\pm 0.2\end{array}$ & $\begin{array}{l}1850.0 \\
\pm 221.0\end{array}$ \\
\hline b & $\begin{array}{c}0.52 \times 0.38 \\
\pm 0.028 \times 0.026\end{array}$ & 5.09 & $\begin{array}{l}95.7 \\
\pm 4.4\end{array}$ & $\begin{array}{c}4089.6 \\
\pm 1.2\end{array}$ & $\begin{array}{l}76.7 \\
\pm 4.4\end{array}$ & 8.9 & $\begin{array}{l}55.3 \\
\pm 2.0\end{array}$ & $\begin{array}{l}13.5 \\
\pm 1.9\end{array}$ & $\begin{array}{l}25.9 \\
\pm 3.6\end{array}$ & $\begin{array}{c}1.7 \\
\pm 0.5\end{array}$ & $\begin{array}{c}7.6 \\
\pm 0.3\end{array}$ & $\begin{array}{l}4084.0 \\
\pm 598.0\end{array}$ \\
\hline $\mathrm{c}$ & $\begin{array}{c}0.72 \times 0.21 \\
\pm 0.025 \times 0.007\end{array}$ & -32.46 & $\begin{array}{l}76.1 \\
\pm 2.1\end{array}$ & $\begin{array}{c}4027.8 \\
\pm 1.9\end{array}$ & $\begin{array}{l}69.1 \\
\pm 5.0\end{array}$ & 9.4 & $\begin{array}{l}41.8 \\
\pm 2.8\end{array}$ & $\begin{array}{l}48.3 \\
\pm 5.9\end{array}$ & $\begin{array}{l}16.0 \\
\pm 2.8\end{array}$ & $\begin{array}{c}6.2 \\
\pm 1.7\end{array}$ & $\begin{array}{c}5.7 \\
\pm 0.4\end{array}$ & $\begin{array}{c}866.0 \\
\pm 120.0\end{array}$ \\
\hline $\mathrm{d}^{\mathrm{a}}$ & $\begin{array}{c}0.94 \times 0.84 \\
\pm 0.016 \times 0.014 \\
\end{array}$ & 0.00 & $\begin{array}{l}182.0 \\
\pm 2.4 \\
\end{array}$ & $\begin{array}{c}4067.3 \\
\pm 1.7 \\
\end{array}$ & $\begin{array}{r}87.3 \\
\pm 4.8 \\
\end{array}$ & 30.2 & $\begin{array}{r}69.4 \\
\pm 5.5 \\
\end{array}$ & $\begin{array}{l}13.0 \\
\pm 3.8\end{array}$ & $\begin{array}{r}66.6 \\
\pm 8.4 \\
\end{array}$ & $\begin{array}{c}1.7 \\
\pm 0.7\end{array}$ & $\begin{array}{c}9.6 \\
\pm 0.8 \\
\end{array}$ & $\begin{array}{c}5320.0 \\
\pm 1603.0 \\
\end{array}$ \\
\hline
\end{tabular}

Note. - The flux shown in this table is measured after the primary beam correction.

Table columns are as follows:

Col. 1: clump number(as Fig. 1a shown).

Col. 2: Major and minor axes by 2-d Gaussian fit.

Col. 3: Major axis position angle (PA; $\mathrm{N}$ to $\mathrm{E})$.

Col. 4: The effective clump radius after a deconvolution with the ALMA beam.

$\left(\mathrm{R}=1.91 \sqrt{\left(\sigma_{x} \times \sigma_{y}\right)}\right.$, where $\sigma=\mathrm{FWHM} / 2.3548$; Solomon et al. 1987)

Col. 5: The line center velocity from 1d Gaussian fit to the line profile within an elliptical aperture with radii of (major and minor axes (FWHM)).

Col. 6: The line velocity FWHM width from 1d Gaussian fit within an elliptical aperture with radii of (major and minor axes (FWHM)).

Col. 7: Clump peak surface brightness from the clump intensity map.

Col. 8: The CO (6-5) flux within an elliptical aperture with radii of (major and minor axes (FWHM)).

Col. 9: The continuum flux density within an elliptical aperture with radii of (major and minor axes (FWHM)).

Col. 10: Virial mass (see the text).

Col. 11: Molecular gas mass (estimated from the dust continuum, see the text).

Col. 12: Molecular gas mass (estimated from the CO flux, see the text).

Col. 13: Ratio of the total CO (6-5) flux to the $435 \mu \mathrm{m}$ dust continuum flux density.

a Using the image in Fig. 3d, corresponding to a larger beam of $0.4^{\prime \prime} \times 0.4^{\prime \prime}$. 